\title{
Multiple-Symbol Differential Sphere Detection and Decision-Feedback Differential Detection Conceived for Differential QAM
}

\author{
Chao Xu, Member, IEEE, Soon Xin Ng, Senior Member, IEEE, and Lajos Hanzo* Fellow, IEEE
}

\begin{abstract}
Multiple-Symbol Differential Sphere Detection (MSDSD) relies on the knowledge of channel correlation. More explicitly, for Differential PSK (DPSK), the transmitted symbols' phases form a unitary matrix, which can be separated from the channel's correlation matrix by the classic Multiple-Symbol Differential Detection (MSDD), so that a lower triangular matrix extracted from the inverted channel correlation matrix is utilized for the MSDSD's sphere decoding. However, for Differential QAM (DQAM), the transmitted symbols' amplitudes cannot form a unitary matrix, which implies that the MSDD's channel correlation matrix becomes amplitude-dependent and remains unknown, unless all the data-carrying symbol amplitudes are detected. In order to tackle this open problem, in this paper, we propose to determine the MSDD's non-constant amplitudedependent channel correlation matrix with the aid of a sphere decoder, so that the classic MSDSD algorithms that were originally conceived for DPSK may also be invoked for DQAM detection. As a result, our simulation results demonstrate that the MSDSD aided DQAM schemes substantially outperform their DPSK counterparts. However, the price paid is that the detection complexity of MSDSD is also significantly increased. In order to mitigate this, we then propose a reduced-complexity MSDSD search strategy specifically conceived for DQAM constellations, which separately map bits to their ring-amplitude index and phase index. Furthermore, the classic Decision-Feedback Differential Detection (DFDD) conceived for DQAM relies on a constant channel correlation matrix, which implies that these DFDD solutions are sub-optimal and they are not equivalent to the optimum MSDD operating in decision-feedback mode. With the advent for solving the open problem of MSDSD aided DQAM, we further propose to improve the conventional DFDD aided DQAM solutions in this paper.
\end{abstract}

Index Terms-Multiple-Symbol Differential Sphere Detection, Decision-Feedback Differential Detection, Differential QAM, Absolute-amplitude Differential Phase Shift Keying, Differential Amplitude Phase Shift Keying, Schnorr-Euchner search strategy.

\section{INTRODUCTION}

$\mathbf{T}$ HE Differential QAM (DQAM) design philosophy was firstly proposed by Weber [1] in 1978, where the phaseambiguity of Star QAM as well as the quadrant-ambiguity of Square QAM incurred by false phase-locking of the carrierrecovery scheme were avoided. More explicitly, Weber [1] applied the Differential PSK (DPSK) principle to the phases of Star QAM symbols, while the ring-amplitudes of the Star QAM symbols were directly transmitted without differential encoding. This scheme was later termed as Absolute-amplitude

The authors are with the School of Electronics and Computer Science, University of Southampton, Southampton SO17 1BJ, UK (e-mail: $\{$ cx1g08,sxn,lh\}@ecs.soton.ac.uk).

The financial support of the European Research Council's Advanced Fellow Grant is gratefully acknowledged.
Differential Phase Shift Keying (ADPSK) by Fischer et al. [2] in order to distinguish it from the family of differentialamplitude DQAM schemes developed later. As a result, the ADPSK receiver invokes a low-complexity noncoherent detector, where the data-carrying phase is recovered by the DPSK scheme's Conventional Differential Detection (CDD), which observes the phase changes between every pair of consecutive received samples, and then the ring-amplitude is demodulated by a quantizer. Similarly, for Square QAM transmission, Weber [1] applied the DPSK scheme's differential encoding to the specific pair of information bits, which determined the particular quadrant, so that the quadrant information was also recovered by the DPSK's CDD at the noncoherent receiver, while the magnitude of the received sample was quantized within the detected quadrant. In 1982, Simon et al. [3] proposed to apply the DPSK scheme's differential encoding philosophy to the Square QAM phase in order to completely eliminate its phase ambiguity problem. However, unlike in PSK and Star QAM, the phase angles of the Square QAM constellation points are not equi-spaced, which inevitably results in arbitrary phase rotations after differential encoding of the phase. For this reason, typically Star QAM constellations are preferred for DQAM design. Furthermore, the classic Differential Amplitude Phase Shift Keying (DAPSK) was proposed by Webb et al. [4] in 1991, where differential encoding was invoked for both the Star QAM ring-amplitude and phase. In 2000, Fischer et al. [2] carried out the capacity comparison of ADPSK, of DAPSK and of their twisted-constellationbased counterparts both in AWGN channels and in quasi-static fading channels. In this paper, the notational form of $M$ $\operatorname{DQAM}\left(M_{A}, M_{P}\right)$ is used for all the DQAM schemes, where $M, M_{A}$ and $M_{P}$ refer to the number of modulation levels, ring-amplitudes and phases, respectively, where we have the relationship of $M=M_{A} M_{P}$.

The development of DQAM has inspired a lot of research. The performance of the CDD aided DAPSK was compared to that of the coherent QAM in [5]-[7], where the DAPSK was shown to be particularly advantageous, when its coherent QAM counterpart suffered from a realistic channel estimation error. However, it was observed in [4] that the performance of CDD aided DAPSK degrades and eventually an error floor is formed in Rayleigh fading channels, when the Doppler frequency is increased. In order to further improve the CDD's performance, the classic Decision-Feedback Differential Detection (DFDD) that was originally proposed for DPSK in [8], [9] was further developed for DAPSK in [10]-[12]. Moreover, the DFDD was also conceived for the absolute-amplitude DQAM schemes in [13]. In more detail, the prediction-based 
DFDD solutions [10]-[13] invoke a low-pass filter in order to estimate the current Channel State Information (CSI) sample based on the previous decisions. Nonetheless, the optimum noncoherent detector for DQAM is the MSDD advocated by Divsalar and Simon [14] in 1994. More explicitly, the MSDD was firstly proposed for DPSK in [15]-[17], where the CDD's observation window width is extended from $N_{w}=2$ to $N_{w} \geq 2$. Consequently, a total of $\left(N_{w}-1\right)$ data-carrying symbols have to be jointly detected. In order to mitigate the MSDD's exponentially increasing complexity, the state-of-theart Multiple-Symbol Differential Sphere Detection (MSDSD) was proposed for DPSK by Lampe et al. [18] in 2005, where the MSDD is facilitated by invoking the Sphere Decoder (SD). The soft-decision MSDSD was further developed for DPSK by Pauli et al. [19] in 2006, so that the MSDSD may be invoked in the context of turbo detection [20].

However, at the time writing, the implementation of MSDD by sphere decoding in the context of DQAM detection is still an open problem, which also has been the most substantial obstacle in the way of offering a solution for MSDSD aided Differential MIMO schemes using the family of bandwidth-efficient QAM constellations [21]-[23]. More explicitly, for DPSK, the transmitted symbols' phases may form a $\left(N_{w} \times N_{w}\right)$-element diagonal matrix $\mathbf{S}$, which is unitary and hence can be separated from the $\left(N_{w} \times N_{w}\right)$-element channel's correlation matrix $\mathbf{C}$ by the classic MSDSD of [18], [19]. By contrast, for the case of MSDD aided DQAM, the $\left(N_{w} \times N_{w}\right)$-element transmitted symbol matrix is given by $\mathbf{S}=\mathbf{A P}$, where $\mathbf{A}$ and $\mathbf{P}$ refer to the $\left(N_{w} \times N_{w}\right)$-element diagonal matrices of transmitted ring-amplitudes and phases, respectively. Naturally, the phase matrix $\mathbf{P}$ is a unitary matrix, but the ring-amplitude matrix $\mathbf{A}$ is not, which cannot be separated from $\mathbf{C}$. As a result, $\mathbf{C}$ remains unknown until all the ring-amplitudes in $\mathbf{A}$ are detected.

Nonetheless, a low-complexity soft-decision MSDD using Iterative Amplitude/Phase processing (MSDD-IAP) was proposed for coded DAPSK in [24], where the MSDD is invoked for ring-amplitude detection, while the MSDSD is employed for phase detection. Then these two detectors iteratively exchange their decisions in order to improve the overall performance. However, the MSDD-IAP still suffers from an error floor in uncoded systems. The reason for this is that without the aid of channel coding, the two detectors may exchange erroneous decisions. Against this background, the novel contributions of this paper are as follows:

1) We observe that although the $\left(N_{w} \times N_{w}\right)$-element channel correlation matrix $\mathbf{C}$ has a total of $M_{A}^{N_{w}}$ candidates, a $(v \times v)$-element partial channel correlation matrix $\widetilde{\mathbf{C}}_{\mathbf{v}}$ that is evaluated with the aid of the SD's previous decisions only has $M_{A}$ candidates according to a single ring-amplitude variable. We prove that the resultant $(v \times v)$-element lower triangular matrix $\widetilde{\mathbf{L}}_{\mathbf{v}}$ that is directly decomposed from $\widetilde{\mathbf{C}}_{\mathbf{v}}^{-1}=\widetilde{\mathbf{L}}_{\mathbf{v}} \widetilde{\mathbf{L}}_{\mathbf{v}}^{H}$ has exactly the same elements as the unkown $\left(N_{w} \times N_{w}\right)$-element lower triangular matrix $\mathbf{L}$ that is decomposed from $\mathbf{C}^{-1}=\mathbf{L L}^{H}$. As a result, the SD may be invoked based on $\left\{\widetilde{\mathbf{L}}_{\mathbf{v}}\right\}_{v=2}^{N_{w}}$ without the knowledge of all DQAM ring- amplitudes. In this way, the classic MSDSD algorithm of [18], [19] may become applicable for all DQAM constellations.

2) Moreover, we propose a reduced-complexity MSDSD search strategy that is explicitly conceived for the DQAM constellations, which separately map the bits to the ring-amplitude index and phase index. Consequently, the complexity imposed by invoking the MSDSD [18], [19] for DQAM may be substantially reduced.

3) Furthermore, the DFDD conceived for DQAM in [12], [13] relied on the assumption of the channel correlation matrix being constant. The resultant DFDD solutions are no longer equivalent to the optimum MSDD of [14] operating in decision-feedback mode. With the advent of solving the open problem of MSDSD aided DQAM in this paper, we propose to improve the conventional DFDD solutions of [12], [13].

4) Finally, we explicitly present the attainable capacity specifically derived for both absolute- and differentialamplitude DQAM constellations in continuous Rayleigh fading channels. The capacity analysis is confirmed by our theoretical and simulation-based BER results, demonstrating that the proposed MSDSD aided DQAM substantially outperforms its DPSK counterpart of [18]. The price paid is that the detection complexity of DQAM relying on $M_{A}$-ring Star QAM constellation is at least about $M_{A}$ times higher than that of its DPSK counterpart, when the proposed hard-decision MSDSD and DFDD are employed.

Without loss of generality, we may readily assume that the OFDM technique and/or interference surpression filters are employed in the scenarios of frequency-selective fading and/or multi-user scenarios, respectively, like within [25]-[27]. As a result, when the proposed MSDSD and DFDD aided DQAM schemes are employed for the sake of mitigating the potential error-floor imposed by a high Doppler frequency, the assumption of having an interference-free flat fading channel model is used.

The rest of this paper is organized as follows. The DQAM constellations are introduced in Sec. II. The MSDD is modelled in the context of DQAM in Sec. III. Furthermore, the MSDSD is conceived for DQAM in Sec. IV. Following this, our improved DFDD solution is designed for DQAM in Sec. V, while our simulation results of capacity, BER and complexity are provided in Sec. VI. Finally, Sec. VII and Sec. VIII offer our conclusions and a discussion of potential future research of turbo detected DQAM, respectively.

The following notations are used throughout the paper. The notations $\ln (\cdot)$ and $\exp (\cdot)$ refer to natural logarithm and natural exponential functions, respectively. The notations $p(\cdot)$ and $\mathrm{E}(\cdot)$ denote the probability and the expectation, respectively. The notations $\Re(\cdot)$ and $\Im(\cdot)$ take the real part and the imaginary part of a complex number, respectively. The operation dec2bin $(\cdot)$ converts a decimal integer to binary bits, while bin2dec $(\cdot)$ converts binary bits to a decimal integer. The operations $(\cdot)^{*},(\cdot)^{T}$ and $(\cdot)^{H}$ denote the conjugate of a complex number, the transpose of a matrix and the Hermitian transpose of a complex matrix, respectively. The operation 


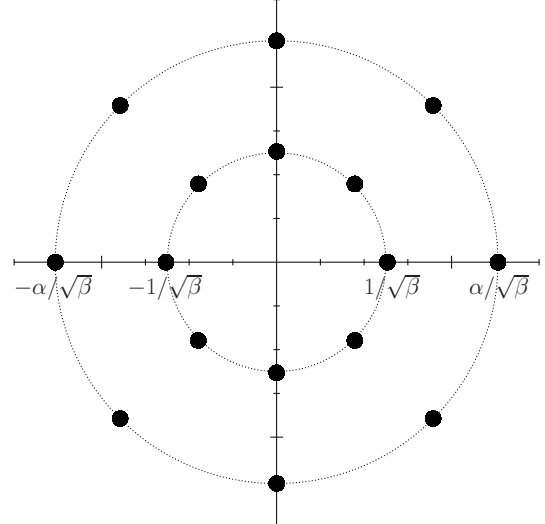

Fig. 1. Constellation diagram of Star 16QAM.

$\otimes$ represents the Kronecker product. The notation $\operatorname{rvec}(\mathbf{A})$ forms a row vector by taking the rows of matrix $\mathbf{A}$ one-byone. Moreover, the operations $\operatorname{diag}\{\mathbf{a}\}$ and $\operatorname{Toeplitz}(\mathbf{a})$ create a diagonal matrix and a symmetric Toeplitz matrix from vector a, respectively.

\section{PREliminaries on DQAM CONSTEllations}

\section{A. Absolute-Amplitude Differential Phase Shift Keying (ADPSK)}

For an $M$-ADPSK $\left(M_{A}, M_{P}\right)$ scheme [1], [2], the transmitter generates a data-carrying Star QAM symbol as:

$$
x^{m}=\gamma^{a} \omega^{p}=\frac{\alpha^{\check{a}}}{\sqrt{\beta}} \exp \left(j \frac{2 \pi}{M_{P}} \check{p}\right),
$$

where the Star QAM modulation index is directly mapped from the BPS $=\log _{2} M$ source bits as $m=$ $\operatorname{bin} 2 \operatorname{dec}\left(b_{1} \cdots b_{\mathrm{BPS}}\right)$. Moreover, the notations of $\gamma^{a}=\frac{\alpha^{a}}{\sqrt{\beta}}$ and $\omega^{p}=\exp \left(j \frac{2 \pi}{M_{P}} \check{p}\right)$ in (1) refer to the ring-amplitude and phase of $x^{m}$, respectively. In more detail, the first $\mathrm{BPS}_{P}=\log _{2} M_{P}$ source bits $\left\{b_{k}\right\}_{k=1}^{\mathrm{BSS}_{P}}$ are assigned to modulate an $M_{P} \mathrm{PSK}$ phase index $p=\operatorname{bin} 2 \operatorname{dec}\left(b_{1} \cdots b_{\mathrm{BPS}_{P}}\right)$, which is the Graycoded index $\check{p}$. The following $\mathrm{BPS}_{A}=\log _{2} M_{A}$ source bits $\left\{b_{k}\right\}_{k=\mathrm{BPS}_{P}+1}^{\mathrm{BPS}}$ are assigned to the $M_{A}$-level ring-amplitude index $a=\operatorname{bin} 2 \operatorname{dec}\left(b_{\mathrm{BPS}_{P}+1} \cdots b_{\mathrm{BPS}}\right)$, which is the Gray coded index $\check{a}$. The relationship between the indices is given by $m=$ $a+p M_{A}$. The notations $\alpha$ and $\beta=\frac{\sum_{\mu=0}^{M_{A}-1} \alpha^{2 \mu}}{M_{A}}$ respectively represent the Star QAM ring ratio and normalization factor. The most advantageous choices for ring ratios in Rayleigh fading channels are $\alpha=2.0$ for twin-ring Star QAM [28], [29] and $\alpha=1.4$ for quadruple-ring Star QAM [30], respectively.

Following (1), the ADPSK transmitter performs differential encoding as [1], [2]:

$$
s_{n}=\frac{1}{\left|s_{n-1}\right|} x_{n-1} s_{n-1},
$$

which starts from $s_{1}=\frac{1}{\sqrt{\beta}}$. Explicitly, the transmitted ADPSK symbols of (2) is represented in the form of $s_{n}=\Gamma_{n} \Omega_{n}$, where $\Gamma_{n}$ and $\Omega_{n}$ refer to the ring-amplitude of and phase of $s_{n}$, respectively. According to (2), the ADPSK scheme invokes differential encoding for the phase as $\Omega_{n}=\omega_{n-1} \Omega_{n-1}$, but no differential encoding is invoked for the ring-amplitude, where the transmitted ADPSK symbols always have the absoluteamplitude of $\Gamma_{n}=\left|s_{n}\right|=\left|x_{n-1}\right|=\gamma_{n-1}$.
Considering the 16-ADPSK $(2,8)$ as an example, the datacarrying symbols $x_{n-1}$ are modulated according to (1) based on the Star 16QAM constellation of Fig. 1. The resultant transmitted symbols $s_{n}$ obtained by the differential encoding of (2) are also drawn from the same Star 16QAM constellation of Fig. 1, thanks to the ring-amplitude normalization of $\frac{1}{\left|s_{n-1}\right|}$ in (2).

\section{B. Differential Amplitude Phase Shift Keying (DAPSK)}

For an $M$-DAPSK $\left(M_{A}, M_{P}\right)$ scheme [4], [31], differential encoding is invoked both for the phase $\Omega_{n}=\omega_{n-1} \Omega_{n-1}$ and for the ring-amplitude $\Gamma_{n}=\gamma_{n-1} \Gamma_{n-1}$ by:

$$
s_{n}=x_{n-1} s_{n-1} \text {, }
$$

which also starts from $s_{1}=\frac{1}{\sqrt{\beta}}$. More explicitly, the transmitted DAPSK symbols $s_{n}$ in (3) are encoded to be Star QAM symbols as [2], [32]:

$$
s_{n}=\Gamma_{n} \Omega_{n}=\frac{\alpha^{\mu_{n}}}{\sqrt{\beta}} \exp \left(j \frac{2 \pi}{M_{P}} q_{n}\right),
$$

where the transmitted symbol's ring-amplitude and phase indices are given by $\left[\mu_{n}=\left(\check{a}+\mu_{n-1}\right) \bmod M_{A}\right]$ and $\left[q_{n}=(\check{p}+\right.$ $\left.\left.q_{n-1}\right) \bmod M_{P}\right]$, respectively, while the data-carrying ringamplitude and phase indices $a=\operatorname{bin} 2 \operatorname{dec}\left(b_{\mathrm{BPS}_{P}+1} \cdots b_{\mathrm{BPS}}\right)$ and $p=\operatorname{bin} 2 \operatorname{dec}\left(b_{1} \cdots b_{\mathrm{BPS}_{P}}\right)$ are Gray coded $\check{a}$ and $\check{p}$, respectively. In this paper, we aim for conceiving generic MSDSD and DFDD schemes, where the DQAM schemes' data-carrying symbols $x_{n-1}$ in (3) have to be detected. Therefore, based on (3) and (4), the modulation of $x_{n-1}$ is formulated as:

$$
x^{m}=\gamma^{a} \omega^{p}=\alpha^{\left\{\left[\left(\check{a}+\mu_{n-1}\right) \bmod M_{A}\right]-\mu_{n-1}\right\}} \cdot \exp \left(j \frac{2 \pi}{M_{P}} \check{p}\right),
$$

where the modulation of the data-carrying ring-amplitude $\gamma^{a}=\alpha^{\left\{\left[\left(\check{a}+\mu_{n-1}\right) \bmod M_{A}\right]-\mu_{n-1}\right\}}$ depends on the previous transmitted ring-amplitude $\Gamma_{n-1}=\frac{\alpha^{\mu_{n-1}}}{\sqrt{\beta}}$, while the datacarrying phase $\omega^{p}=\exp \left(j \frac{2 \pi}{M_{P}} \check{p}\right)$ is directly modulated as $M_{P}$ PSK.

Let us consider the 16-DAPSK $(2,8)$ as an example, which encodes $s_{n}=\Gamma_{n} \Omega_{n}$ of (4) according to the Star 16QAM constellation of Fig. 1. More explicitly, the first three bits are assigned to an 8PSK phase $\omega_{n-1}$, and then the transmitted phase obtained by $\Omega_{n}=\omega_{n-1} \Omega_{n-1}$ is drawn from the same 8PSK phase set, as seen in Fig. 1. Moreover, according to (5), when we have $\mu_{n-1}=0$ and hence $\Gamma_{n-1}=\frac{1}{\sqrt{\beta}}$, the last source bit $b_{4} \in\{0,1\}$ is mapped to $\gamma_{n-1} \in\left\{\alpha^{\breve{a}}\right\}_{\breve{a}=0}^{1}=\{1, \alpha\}$. Similarly, when we have $\mu_{n-1}=1$ and hence $\Gamma_{n-1}=\frac{\alpha}{\sqrt{\beta}}, b_{4} \in\{0,1\}$ is mapped to $\gamma_{n-1} \in\left\{\alpha^{\{[(\breve{a}+1) \bmod 2]-1\}}\right\}_{\breve{a}=0}^{1}=\left\{1, \frac{1}{\alpha}\right\}$. As a result, the transmitted ring-amplitude obtained by $\Gamma_{n}=\gamma_{n-1} \Gamma_{n-1}$ is always drawn from the twin-ring set of $\left\{\frac{1}{\sqrt{\beta}}, \frac{\alpha}{\sqrt{\beta}}\right\}$, as seen in Fig. 1.

\section{Twisted ADPSK (TADPSK) and Twisted DAPSK (TDAPSK)}

As proposed in [2], [13], a ring-amplitude-dependent phase rotation is capable of increasing the Star QAM constellation distances. Let us firstly consider a generic $M$ $\operatorname{TADPSK}\left(M_{A}, M_{P}\right)$. The differential encoding of TADPSK is 
the same as that of ADPSK as specified by (2). However, the TADPSK data-carrying symbol $x_{n-1}=\gamma_{n-1} \omega_{n-1} \psi_{n-1}$ contains the extra phase rotation term of $\psi_{n-1}$, which is determined by the ring-amplitude index $a$ as given by $\psi^{a}=$ $\exp \left(j \frac{2 \pi}{M} \check{a}\right)$. Therefore, the modulation of the TADPSK's datacarrying symbol $x_{n-1}$ is now given by:

$$
x^{m}=\gamma^{a} \omega^{p} \psi^{a}=\frac{\alpha^{\check{a}}}{\sqrt{\beta}} \exp \left(j \frac{2 \pi}{M_{P}} \check{p}\right) \exp \left(j \frac{2 \pi}{M} \check{a}\right) .
$$

As a result, the TADPSK transmitted symbol $s_{n}$ according to (2) also contains an extra phase rotation term $\Psi_{n}$, i.e. we have $s_{n}=\Gamma_{n} \Omega_{n} \Psi_{n}$, where the differential encoding process is performed on both the phase $\Omega_{n}=\omega_{n-1} \Omega_{n-1}$ and on the ring-amplitude-dependent phase rotation $\Psi_{n}=\psi_{n-1} \Psi_{n-1}$, but we still have the absolute-amplitude of $\Gamma_{n}=\left|s_{n}\right|=$ $\left|x_{n-1}\right|=\gamma_{n-1}$.

Similarly, the TDAPSK is obtained by twisting the DAPSK constellation. The differential encoding process of TDAPSK is the same as that of DAPSK formulated by (3). However, the modulation of the TDAPSK data-carrying symbol $x^{m}=$ $\gamma^{a} \omega^{p} \psi^{a}$ is modified as:

$$
x^{m}=\alpha^{\left\{\left[\left(\check{a}+\mu_{n-1}\right) \bmod M_{A}\right]-\mu_{n-1}\right\}} \exp \left(j \frac{2 \pi}{M_{P}} \check{p}\right) \exp \left(j \frac{2 \pi}{M} \check{a}\right) .
$$

Therefore, the TDAPSK transmitted symbol is also represented by $s_{n}=\Gamma_{n} \Omega_{n} \Psi_{n}$, where the differential encoding of (3) results in $\Gamma_{n}=\gamma_{n-1} \Gamma_{n-1}, \Omega_{n}=\omega_{n-1} \Omega_{n-1}$ and $\Psi_{n}=\psi_{n-1} \Psi_{n-1}$.

Moreover, all the aforementioned DQAM constellations separately modulate the ring-amplitude and phase. By contrast, it was introduced in [2], [13] that the two terms may be jointly modulated, which is represented in the form of DQAM ${ }^{\mathrm{JM}}$. For example, the joint mapping conceived for the TADPSK constellation of (6) is expressed as:

$$
x^{m}=\frac{\alpha^{\left(\check{m} \bmod M_{A}\right)}}{\sqrt{\beta}} \exp \left(j \frac{2 \pi}{M} \check{m}\right),
$$

where all the BPS $=\log _{2} M$ source bits are assigned to encode the global modulation index of $m=\operatorname{bin} 2 \operatorname{dec}\left(b_{1} \cdots b_{\mathrm{BPS}}\right)$, which is the Gray coded index $\check{m}$. The resultant constellation is referred to as TADPSK ${ }^{\mathrm{JM}}$. Similarly, the joint mapping designed for TDAPSK constellation of (7) is formulated as:

$$
x^{m}=\frac{\alpha^{\left\{\left[\left(\check{m} \bmod M_{A}\right)+\mu_{n-1}\right] \bmod M_{A}\right\}}}{\alpha^{\mu_{n-1}}} \exp \left(j \frac{2 \pi}{M} \check{m}\right),
$$

which is referred to as TDAPSK ${ }^{\mathrm{JM}}$. It is worthy to note that DQAM and its DQAM ${ }^{\mathrm{JM}}$ counterpart which have the same constellation topology achieve the same capacity. The related examples for twisted DQAM and DQAM ${ }^{\mathrm{JM}}$ may be found in [2], [13].

In summary, the DQAM constellations discussed in this paper include the absolute-amplitude DQAM schemes of ADPSK/TADPSK/TADPSK ${ }^{\mathrm{JM}}$, which invoke the differential encoding process of (2), as well as the differential-amplitude DQAM instantiations of DAPSK/TDAPSK/TDAPSK ${ }^{\mathrm{JM}}$, which rely on the differential encoding process of (3).

\section{Multiple-Symbol Differential Detection}

For a Single-Input Multiple-Output (SIMO) system, the signals received by the $N_{R}$ receive antennas are modelled as $\mathbf{Y}_{n}=s_{n} \mathbf{H}_{n}+\mathbf{V}_{n}$, where the $N_{R}$-element row vectors $\mathbf{Y}_{n}$, $\mathbf{H}_{n}$ and $\mathbf{V}_{n}$ model the received signal, the Rayleigh fading and the AWGN, respectively. The MSDD models the received signal as ${ }^{1}$ :

$$
\mathbf{Y}=\mathbf{S H}+\mathbf{V}=\mathbf{A P O H}+\mathbf{V}
$$

where the received signal matrix $\mathbf{Y}=$ $\left[\mathbf{Y}_{N_{w}}^{T}, \mathbf{Y}_{N_{w}-1}^{T}, \cdots, \mathbf{Y}_{1}^{T}\right]^{T}$, the fading channel matrix $\mathbf{H}=\left[\mathbf{H}_{N_{w}}^{T}, \mathbf{H}_{N_{w}-1}^{T}, \cdots, \mathbf{H}_{1}^{T}\right]^{T}$ and the AWGN matrix $\mathbf{V}=\left[\mathbf{V}_{N_{w}}^{T_{w}}, \mathbf{V}_{N_{w}-1}^{T}, \cdots, \mathbf{V}_{1}^{T}\right]^{T}$ are of size $\left(N_{w} \times N_{R}\right)$. Moreover, the transmitted symbol matrix $\mathbf{S}=\operatorname{diag}\left\{\left[s_{N_{w}}, s_{N_{w}-1}, \cdots, s_{1}\right]\right\}$, the amplitude matrix $\mathbf{A}=\operatorname{diag}\left\{\left[\Gamma_{N_{w}}, \Gamma_{N_{w}-1}, \cdots, \Gamma_{1}\right]\right\}$, the phase matrix $\mathbf{P}=\operatorname{diag}\left\{\left[\Omega_{N_{w}}, \Omega_{N_{w}-1}, \cdots, \Omega_{1}\right]\right\}$ and the ring-amplitude-dependent phase rotation matrix $\mathbf{O}=\operatorname{diag}\left\{\left[\Psi_{N_{w}}, \Psi_{N_{w}-1}, \cdots, \Psi_{1}\right]\right\}$ are all of size $\left(N_{w} \times N_{w}\right)$. We note that $\mathbf{O}$ is an identity matrix for ADPSK and DAPSK. Moreover, both $\Omega_{1}$ and $\Psi_{1}$ are common phase rotations of the following symbols. Hence they should be separated from $\mathbf{P}$ and $\mathbf{O}$ in (10), which leads to:

$$
\mathbf{Y}=\overline{\mathbf{A}} \overline{\mathbf{P}} \overline{\mathbf{H}} \overline{\mathbf{H}}+\mathbf{V},
$$

where the $v^{\text {th }}$ diagonal element in $\overline{\mathbf{P}}$ is given by $\bar{\Omega}_{v}=\Omega_{v} \Omega_{1}^{*}$, which leads to $\bar{\Omega}_{1}=1$ and $\bar{\Omega}_{v}=\omega_{v-1} \bar{\Omega}_{v-1}=\prod_{t=1}^{v-1} \omega_{t}$ for $v>1$. Similarly, the $v^{\text {th }}$ diagonal element in $\overline{\mathbf{O}}$ is given by $\bar{\Psi}_{v}=\Psi_{v} \Psi_{1}^{*}$, which leads to $\bar{\Psi}_{1}=1$ and $\bar{\Psi}_{v}=$ $\psi_{v-1} \bar{\Psi}_{v-1}=\prod_{t=1}^{v-1} \psi_{t}$ for $v>1$. As a result, the $v^{t h}$ row in $\overline{\mathbf{H}}$ is given by $\mathbf{H}_{v}=\Omega_{1} \Psi_{1} \mathbf{H}_{v}$. However, unlike $\Omega_{1}$ and $\Psi_{1}$, the value of $\Gamma_{1}$ does affect the MSDD decision, but $\Gamma_{1}$ does not carry source information for the current MSDD window. Therefore, when $\overline{\mathbf{A}}$ in (10) is detected by the MSDD, $\Gamma_{1}$ is considered as a known term, which is either obtained based on previous MSDD decisions or detected separately as an unknown variable. As a result, there are $M_{A}^{\left(N_{w}-1\right)}$ combinations for $\overline{\mathbf{A}}$ in (10). Specifically, for the absoluteamplitude ADPSK/TADPSK/TADPSK ${ }^{\mathrm{JM}}$ using (2), the $v^{\text {th }}$ diagonal element in $\overline{\mathbf{A}}$ is given by $\Gamma_{v}=\gamma_{v-1}$. By contrast, for the differential-amplitude DAPSK/TDAPSK/TDAPSK ${ }^{\mathrm{JM}}$ using (3), we have $\Gamma_{v}=\gamma_{v-1} \Gamma_{v-1}=\left(\prod_{t=1}^{v-1} \gamma_{t}\right) \Gamma_{1}$.

The MSDD aims for maximizing the following a posteriori probability:

$$
p(\overline{\mathbf{A}}, \overline{\mathbf{P}} \mid \mathbf{Y})=\sum_{\forall \Gamma_{1}} \frac{p\left(\mathbf{Y} \mid \overline{\mathbf{A}}, \overline{\mathbf{P}}, \Gamma_{1}\right) p(\overline{\mathbf{A}}) p(\overline{\mathbf{P}})}{\sum_{\forall \overline{\mathbf{A}}, \forall \overline{\mathbf{P}}} p\left(\mathbf{Y} \mid \overline{\mathbf{A}}, \overline{\mathbf{P}}, \Gamma_{1}\right) p(\overline{\mathbf{A}}) p(\overline{\mathbf{P}})} p\left(\Gamma_{1}\right),
$$

where $p\left(\Gamma_{1}\right), p(\overline{\mathbf{A}})$ and $p(\overline{\mathbf{P}})$ refer to the a priori probabilities of $\Gamma_{1}, \overline{\mathbf{A}}$ and $\overline{\mathbf{P}}$, respectively, which may all be assumed to be equiprobable in uncoded systems. Furthermore, according to (11), the probability of receiving $\mathbf{Y}$ given $\overline{\mathbf{A}}, \overline{\mathbf{P}}$ and $\Gamma_{1}$ is

${ }^{1} \mathbf{Y}$ in (10) stores received signal vectors in a reverse order compared to [18], [19], so that the MSDSD may detect the phases according to their differential encoding order of $\Omega_{v}=\omega_{v-1} \Omega_{v-1}$, instead of detecting them backwards as $\Omega_{v-1}=\omega_{v-1}^{*} \Omega_{v}$. 
formulated as [14], [17], [18]:

$$
p\left(\mathbf{Y} \mid \overline{\mathbf{A}}, \overline{\mathbf{P}}, \Gamma_{1}\right)=\frac{\exp \left\{-\operatorname{rvec}(\mathbf{Y}) \cdot \mathbf{R}_{Y Y}^{-1}[\operatorname{rvec}(\mathbf{Y})]^{H}\right\}}{\pi^{N_{R} N_{w}} \operatorname{det}\left(\mathbf{R}_{Y Y}\right)},
$$

where the equivalent MSDD received signal model of (11) becomes $\operatorname{rvec}(\mathbf{Y})=\operatorname{rvec}(\overline{\mathbf{H}}) \cdot\left[(\overline{\mathbf{A}} \overline{\mathbf{P}} \overline{\mathbf{O}}) \otimes \mathbf{I}_{N_{R}}\right]+\operatorname{rvec}(\mathbf{V})$, where the operation $\otimes$ represents the Kronecker product. As a result, the correlation matrix seen in (13) is expressed as:

$\mathbf{R}_{Y Y}=\mathrm{E}\left\{[\operatorname{rvec}(\mathbf{Y})]^{H} \cdot \operatorname{rvec}(\mathbf{Y})\right\}=\left(\overline{\mathbf{O}}^{H} \overline{\mathbf{P}}^{H} \mathbf{C} \overline{\mathbf{P}} \overline{\mathbf{O}}\right) \otimes \mathbf{I}_{N_{R}}$,

where both $\overline{\mathbf{P}}$ and $\overline{\mathbf{O}}$ are unitary matrices. Moreover, the channel's characteristic correlation matrix $\mathbf{C}$ seen in (14) is given by:

$$
\mathbf{C}=\overline{\mathbf{A}}^{H} \mathbf{R}_{h h} \overline{\mathbf{A}}+\mathbf{R}_{v v},
$$

where the fading characteristic matrix $\mathbf{R}_{h h}=$ $\operatorname{Toeplitz}\left(\left[\begin{array}{llll}\rho_{0} & \rho_{1} & \cdots & \rho_{N_{w}-1}\end{array}\right]\right)$ and the AWGN characteristic matrix $\mathbf{R}_{v v}=N_{0} \mathbf{I}_{\mathbf{N}_{\mathrm{w}}}$ are the same as in the case of DPSK using $N_{R}=1$ in [18], [19]. More explicitly, the temporal correlation between the fading factors is defined by $\left\{\rho_{v}=J_{0}\left(2 \pi f_{d} \cdot v\right)\right\}_{v=0}^{N_{w}-1}$ according to Clarke's [33] fading model, where $J_{0}(\cdot)$ is the zero-order Bessel function of the first kind, while $f_{d}=\frac{v \cdot f_{c}}{c \cdot f_{s}}$ denotes the normalized Doppler frequency, as $v, f_{c}, c$ and $f_{s}$ refer to the velocity of the mobile receiver, the carrier frequency, the speed of light and the sampling rate, respectively.

However, since $\overline{\mathbf{A}}$ is not a unitary matrix, it cannot be separated from $\mathbf{C}$ in (15). As a result, in contrast to the case of DPSK in [18], [19], $\overline{\mathbf{A}}^{H} \mathbf{R}_{h h} \overline{\mathbf{A}}$ seen in (15) is neither a constant matrix nor a Toeplitz matrix. This implies that $\mathbf{C}$ of (15) does not become known until all the ring-amplitudes in $\overline{\mathbf{A}}$ are detected. In summary, the MSDD that maximizes (12) is formulated as:

$$
\begin{array}{r}
\{\hat{\overline{\mathbf{A}}}, \hat{\overline{\mathbf{P}}}\}=\max _{\forall \Gamma_{1}} \max _{\forall \overline{\mathbf{A}}, \forall \overline{\mathbf{P}}}-\operatorname{tr}\left(\mathbf{Y}^{H} \overline{\mathbf{P}} \overline{\mathbf{O}} \mathbf{C}^{-1} \overline{\mathbf{O}}^{H} \overline{\mathbf{P}}^{H} \mathbf{Y}\right) \\
-N_{R} \ln [\operatorname{det}(\mathbf{C})],
\end{array}
$$

where the determinant in (13) is given by $\operatorname{det}\left(\mathbf{R}_{Y Y}\right)=$ $\operatorname{det}(\mathbf{C})^{N_{R}}$. Furthermore, if $\hat{\Gamma}_{1}$ is fed back from the previous MSDD decisions, then a Hard-Decision-Directed MSDD (HDD-MSDD) is simply formulated as:

$$
\{\hat{\overline{\mathbf{A}}}, \hat{\overline{\mathbf{P}}}\}=\max _{\forall \overline{\mathbf{A}}, \forall \overline{\mathbf{P}}}-\operatorname{tr}\left(\mathbf{Y}^{H} \overline{\mathbf{P}} \overline{\mathbf{O}} \mathbf{C}^{-1} \overline{\mathbf{O}}^{H} \overline{\mathbf{P}}^{H} \mathbf{Y}\right)-N_{R} \ln [\operatorname{det}(\mathbf{C})] .
$$

Then the newly detected $\hat{\Gamma}_{N_{w}}$ in $\hat{\overline{\mathbf{A}}}$ may be passed on to the next MSDD window as $\Gamma_{1}$. We note that the absoluteamplitude DQAM of ADPSK/TADPSK/TADPSK ${ }^{\mathrm{JM}}$ can only employ the HDD-MSDD of (17). Let us consider MSDD aided ADPSK associated with $N_{w}=2$ as an example, where the decision in (16) becomes $\min _{\forall \Gamma_{1}} \min _{\forall \gamma_{1}, \omega_{1}}\left\{\frac{1}{\operatorname{det}(\mathbf{C})}\left[\left(\Gamma_{2}^{2}+\right.\right.\right.$ $\left.\left.N_{0}\right)\left\|\mathbf{Y}_{1}\right\|^{2}+\left(\Gamma_{1}^{2}+N_{0}\right)\left\|\mathbf{Y}_{2}\right\|^{2}-2 \Gamma_{1} \Gamma_{2} \rho_{1} \Re\left(\omega_{1}^{*} \mathbf{Y}_{2} \mathbf{Y}_{1}^{H}\right)\right]+$ $\left.N_{R} \ln [\operatorname{det}(\mathbf{C})]\right\}$, while the determinant term $\left[\operatorname{det}(\mathbf{C})=\left(\Gamma_{1}^{2}+\right.\right.$ $\left.\left.N_{0}\right)\left(\Gamma_{2}^{2}+N_{0}\right)-\Gamma_{1}^{2} \Gamma_{2}^{2} \rho_{1}^{2}\right]$ tends to zero, as the SNR increases. This leads us to the ADPSK decision of $\min _{\forall \Gamma_{1}} \min _{\forall \gamma_{1}, \omega_{1}} \Gamma_{1}^{2}$. $\left\|\mathbf{Y}_{2}-\frac{\Gamma_{2}}{\Gamma_{1}} \omega_{1} \mathbf{Y}_{1}\right\|^{2}$ for the simplified situation of $\rho_{1} \approx 1$. This implies that if both $\Gamma_{1}$ and $\Gamma_{2}=\gamma_{1}$ are variables for the ADPSK detection, both the case of $\left[\Gamma_{1}=\frac{1}{\sqrt{\beta}}, \gamma_{1}=\frac{1}{\sqrt{\beta}}\right]$ and the case of $\left[\Gamma_{1}=\frac{\alpha}{\sqrt{\beta}}, \gamma_{1}=\frac{\alpha}{\sqrt{\beta}}\right]$ would be detected as $\left[\Gamma_{1}=\frac{1}{\sqrt{\beta}}, \gamma_{1}=\frac{1}{\sqrt{\beta}}\right]$, which imposes ambiguity, because $\gamma_{1}=\frac{1}{\sqrt{\beta}}$ and $\gamma_{1}=\frac{\alpha}{\sqrt{\beta}}$ carry different source information. We note that this is not a problem for DAPSK, because both the cases of $\left[\Gamma_{1}=\Gamma_{2}=\frac{1}{\sqrt{\beta}}\right]$ and $\left[\Gamma_{1}=\Gamma_{2}=\frac{\alpha}{\sqrt{\beta}}\right]$ carry the same information for $\gamma_{1}=\Gamma_{2} / \Gamma_{1}$ according to (3).

\section{Multiple-Symbol Differential Sphere DETECTION}

In order to invoke SD for MSDD aided DQAM, we firstly have to reformulate the MSDD metric of (16) as a summation of increments, so that the SD becomes capable of evaluating a single metric at a time. Secondly, the Schnorr-Euchner search strategy of [34] should be tailored for MSDSD aided DQAM. Thirdly, a reduced-complexity search strategy is proposed for the MSDSD of DQAM schemes that separately modulate their ring-amplitude and phase.

\section{A. Partial Euclidean Distance (PED)}

First of all, the MSDD of (16) is rewritten in form of Euclidean Distance (ED) as:

$\{\hat{\overline{\mathbf{A}}}, \hat{\overline{\mathbf{P}}}\}=\min _{\forall \Gamma_{1}}\left\{\min _{\forall \overline{\mathbf{A}}, \forall \overline{\mathbf{P}}}\left\|\mathbf{L}^{T} \overline{\mathbf{O}}^{H} \overline{\mathbf{P}}^{H} \mathbf{Y}\right\|^{2}+N_{R} \cdot \ln [\operatorname{det}(\mathbf{C})]\right\}$,

where we have the trace function property of $\operatorname{tr}\left(\mathbf{B}^{H} \mathbf{B}\right)=$ $\|\mathbf{B}\|^{2}$, while the lower triangular matrix $\mathbf{L}$ is derived from decomposition of $\mathbf{C}^{-1}=\mathbf{L} \mathbf{L}^{T}$. We note that $\mathbf{L}$ and $\ln [\operatorname{det}(\mathbf{C})]$ of (18) remain unknown, until the entire ring-amplitude matrix $\overline{\mathbf{A}}$ is detected. In order to solve this problem by a sphere decoder, we conceive two propositions as follows:

Proposition 1: The first term in the ED of (18) may be extended as:

$$
\begin{aligned}
\left\|\mathbf{L}^{T} \overline{\mathbf{O}}^{H} \overline{\mathbf{P}}^{H} \mathbf{Y}\right\|^{2}=\left\|l_{N_{w}, N_{w}} \mathbf{Y}_{1}\right\|^{2} & \\
& +\sum_{v=2}^{N_{w}}\left\|\sum_{t=1}^{v} l_{N_{w}-t+1, N_{w}-v+1} \bar{\Psi}_{t}^{*} \bar{\Omega}_{t}^{*} \mathbf{Y}_{t}\right\|^{2},
\end{aligned}
$$

where the coefficients $\left\{\left\{l_{N_{w}-t+1, N_{w}-v+1}\right\}_{t=1}^{v}\right\}_{v=1}^{N_{w}}$ are elements in $\mathbf{L}$. It can be seen in (19) that for a specific index $v$, only a subset of the coefficients $\left\{l_{N_{w}-t+1, N_{w}-v+1}\right\}_{t=1}^{v}$ from a $(v \times v)$-element submatrix $\widetilde{\mathbf{L}}_{\mathbf{v}}$ is required, where $\mathbf{L}$ in (19) is expressed in the form of submatrices as:

$$
\mathbf{L}=\left[\begin{array}{ll}
\widetilde{\mathbf{E}}_{\mathbf{v}} & \mathbf{0}_{N_{w}-v, v} \\
\widetilde{\mathbf{G}}_{\mathbf{v}} & \widetilde{\mathbf{L}}_{\mathbf{v}}
\end{array}\right] .
$$

The submatrices $\widetilde{\mathbf{E}}_{\mathbf{v}}$ and $\widetilde{\mathbf{G}}_{\mathbf{v}}$ are of size $\left(N_{w}-v\right) \times\left(N_{w}-v\right)$ and $v \times\left(N_{w}-v\right)$, respectively, while the all-zero submatrix $\mathbf{0}_{N_{w}-v, v}$ are of size $\left(N_{w}-v\right) \times v$. We will formally show below that although $\mathbf{C}$ and $\mathbf{L}$ are unknown, $\widetilde{\mathbf{L}}_{\mathbf{v}}$ may be obtained by the Cholesky decomposition $\widetilde{\mathbf{L}}_{\mathbf{v}} \widetilde{\mathbf{L}}_{\mathbf{v}}^{T}=\widetilde{\mathbf{C}}_{\mathbf{v}}^{-1}$, where the partial channel correlation matrix $\widetilde{\mathbf{C}}_{\mathbf{v}}$ may be evaluated with the aid of the SD's previous decisions concerning $\left\{\Gamma_{t}\right\}_{t=1}^{v-1}$ 
and a single variable $\Gamma_{v}$ as:

$$
\begin{aligned}
\widetilde{\mathbf{C}}_{\mathbf{v}} & =\left[\begin{array}{cccc}
\Gamma_{v}^{2} \rho_{0}+N_{0} & \Gamma_{v} \Gamma_{v-1} \rho_{1} & \cdots & \Gamma_{v} \Gamma_{1} \rho_{v-1} \\
\Gamma_{v-1} \Gamma_{v} \rho_{1} & \Gamma_{v-1}^{2} \rho_{0}+N_{0} & \cdots & \Gamma_{v-1} \Gamma_{1} \rho_{v-2} \\
\vdots & \vdots & \ddots & \vdots \\
\Gamma_{1} \Gamma_{v} \rho_{v-1} & \Gamma_{1} \Gamma_{v-1} \rho_{v-2} & \cdots & \Gamma_{1}^{2} \rho_{0}+N_{0}
\end{array}\right] \\
& =\left[\begin{array}{cc}
\Gamma_{v}^{2} \rho_{0}+N_{0} & \widetilde{\mathbf{e}}_{v}^{T} \\
\widetilde{\mathbf{e}}_{v} & \widetilde{\mathbf{C}}_{\mathbf{v}-\mathbf{1}}
\end{array}\right] .
\end{aligned}
$$

The $(v-1)$-element column vector $\widetilde{\mathbf{e}}_{v}$ in (21) is given by $\widetilde{\mathbf{e}}_{v}=\left[\Gamma_{v} \Gamma_{v-1} \rho_{1}, \cdots, \Gamma_{v} \Gamma_{1} \rho_{v-1}\right]^{T}$.

Proof: Similar to $\mathbf{L}$ expressed in (20), the Hermitian matrix $\mathbf{C}$ of (15) may be expressed in the form of submatrices as:

$$
\mathbf{C}=\left[\begin{array}{ll}
\widetilde{\mathbf{B}}_{\mathbf{v}} & \widetilde{\mathbf{D}}_{\mathbf{v}}^{T} \\
\widetilde{\mathbf{D}}_{\mathbf{v}} & \widetilde{\mathbf{C}}_{\mathbf{v}}
\end{array}\right]
$$

where $\widetilde{\mathbf{B}}_{\mathbf{v}}$ and $\widetilde{\mathbf{D}}_{\mathbf{v}}$ are of size $\left(N_{w}-v\right) \times\left(N_{w}-v\right)$ and $v \times\left(N_{w}-v\right)$, respectively, while $\widetilde{\mathbf{C}}_{\mathbf{v}}$ was defined in (21). According to the blockwise matrix inversion property [35], the matrix inverse $\mathbf{C}^{-1}$ is expressed as:

$$
\mathbf{C}^{-1}=\left[\begin{array}{ll}
\widetilde{\mathbf{Q}}_{\mathbf{v}} & -\widetilde{\mathbf{Q}}_{\mathbf{v}} \widetilde{\mathbf{D}}_{\mathbf{v}}^{T} \widetilde{\mathbf{C}}_{\mathbf{v}}^{-1} \\
-\widetilde{\mathbf{C}}_{\mathbf{v}}^{-1} \widetilde{\mathbf{D}}_{\mathbf{v}} \widetilde{\mathbf{Q}}_{\mathbf{v}} & \widetilde{\mathbf{C}}_{\mathbf{v}}^{-1} \widetilde{\mathbf{D}}_{\mathbf{v}} \widetilde{\mathbf{Q}}_{\mathbf{v}} \widetilde{\mathbf{D}}_{\mathbf{v}}^{T} \widetilde{\mathbf{C}}_{\mathbf{v}}^{-1}+\widetilde{\mathbf{C}}_{\mathbf{v}}^{-1}
\end{array}\right],
$$

where $\widetilde{\mathbf{Q}}_{\mathbf{v}}=\left(\widetilde{\mathbf{B}}_{\mathbf{v}}-\widetilde{\mathbf{D}}_{\mathbf{v}}^{T} \widetilde{\mathbf{C}}_{\mathbf{v}}^{-1} \widetilde{\mathbf{D}}_{\mathbf{v}}\right)^{-1}$ is a Hermitian matrix. According to $\mathbf{L L}^{T}=\mathbf{C}^{-1}$, we have the following relationships based on (20) and (23): $\widetilde{\mathbf{E}}_{\mathbf{v}} \widetilde{\mathbf{E}}_{\mathbf{v}}^{T}=\widetilde{\mathbf{Q}}_{\mathbf{v}}, \widetilde{\mathbf{G}}_{\mathbf{v}} \widetilde{\mathbf{E}}_{\mathbf{v}}^{T}=$ $\widetilde{\mathbf{C}}_{\mathbf{v}}^{-1} \widetilde{\mathbf{D}}_{\mathbf{v}} \widetilde{\mathbf{Q}}_{\mathbf{v}}$ and $\widetilde{\mathbf{G}}_{\mathbf{v}} \widetilde{\mathbf{G}}_{\mathbf{v}}^{T}+\widetilde{\mathbf{L}}_{\mathbf{v}} \widetilde{\mathbf{L}}_{\mathbf{v}}^{T}=\widetilde{\mathbf{C}}_{\mathbf{v}}^{-1} \widetilde{\mathbf{D}}_{\mathbf{v}} \widetilde{\mathbf{Q}}_{\mathbf{v}} \widetilde{\mathbf{D}}_{\mathbf{v}}^{T} \widetilde{\mathbf{C}}_{\mathbf{v}}^{-1}+$ $\widetilde{\mathbf{C}}_{\mathrm{v}}^{-1}$, which leads to the following conclusions $\widetilde{\mathbf{G}}_{\mathbf{v}}=$ $-\mathbf{C}_{\mathbf{y}}^{-1} \widetilde{\mathbf{D}}_{\mathbf{v}} \widetilde{\mathbf{E}}_{\mathbf{v}}, \widetilde{\mathbf{G}}_{\mathbf{v}} \widetilde{\mathbf{G}}_{\mathbf{v}}^{T}=\widetilde{\mathbf{C}}_{\mathbf{y}}^{-1} \widetilde{\mathbf{D}}_{\mathbf{v}} \widetilde{\mathbf{Q}}_{\mathbf{v}} \widetilde{\mathbf{D}}_{\mathbf{v}}^{T} \widetilde{\mathbf{C}}_{\mathbf{v}}^{-1}$ and then finally $\widetilde{\mathbf{L}}_{\mathbf{v}} \widetilde{\mathbf{L}}_{\mathbf{v}}^{T}=\widetilde{\mathbf{C}}_{\mathbf{v}}^{-1}$. Therefore, $\widetilde{\mathbf{L}}_{\mathbf{v}}$ in (20) may be directly obtained from the Cholesky decomposition of $\widetilde{\mathbf{C}}_{\mathbf{v}}^{-1}$.

Proposition 2: We propose to evaluate the second term in the ED of (18) by:

$$
\begin{array}{r}
\ln [\operatorname{det}(\mathbf{C})]=\ln \left(\Gamma_{1}^{2} \rho_{0}+N_{0}\right)+\sum_{v=2}^{N_{w}} \ln \left[\left(\Gamma_{v}^{2} \rho_{0}+N_{0}\right)\right. \\
\left.-\widetilde{\mathbf{e}}_{v}^{T} \widetilde{\mathbf{C}}_{\mathbf{v}-\mathbf{1}}^{-1} \widetilde{\mathbf{e}}_{v}\right] .
\end{array}
$$

Proof: According to the Leibniz formula [35], the determinant of $\widetilde{\mathbf{C}}_{\mathbf{v}}$ in (21) may be evaluated by $\operatorname{det}\left(\widetilde{\mathbf{C}}_{\mathbf{v}}\right)=$ $\operatorname{det}\left(\widetilde{\mathbf{C}}_{\mathbf{v}-\mathbf{1}}\right)\left[\left(\Gamma_{v}^{2} \rho_{0}+N_{0}\right)-\widetilde{\mathbf{e}}_{v}^{T} \widetilde{\mathbf{C}}_{\mathbf{v}-\mathbf{1}}^{-1} \widetilde{\mathbf{e}}_{v}\right]$. This implies that the evaluation of $\operatorname{det}\left(\widetilde{\mathbf{C}}_{\mathbf{v}}\right)$ may be carried out in the logarithmic domain by adding an incremental term to the previous evaluation as follows $\ln \operatorname{det}\left(\widetilde{\mathbf{C}}_{\mathbf{v}}\right)=\ln \operatorname{det}\left(\widetilde{\mathbf{C}}_{\mathbf{v}-\mathbf{1}}\right)+\ln \left[\left(\Gamma_{v}^{2} \rho_{0}+\right.\right.$ $\left.\left.N_{0}\right)-\widetilde{\mathbf{e}}_{v}^{T} \widetilde{\mathbf{C}}_{\mathbf{v}-1}^{-1} \widetilde{\mathbf{e}}_{v}\right]$. Therefore, the complete determinant term $\ln [\operatorname{det}(\mathbf{C})]$ may be calculated by a SD from the initial term that is associated with the index of $v=1$ as $\ln \left[\operatorname{det}\left(\widetilde{\mathbf{C}}_{\mathbf{1}}\right)\right]=$ $\ln \left(\Gamma_{1}^{2} \rho_{0}+N_{0}\right)$, in addition to the summation of all incremental terms $\sum_{v=2}^{N_{w}} \ln \left[\left(\Gamma_{v}^{2} \rho_{0}+N_{0}\right)-\widetilde{\mathbf{e}}_{v}^{T} \widetilde{\mathbf{C}}_{\mathbf{v}-1}^{-1} \widetilde{\mathbf{e}}_{v}\right]$.

As a result, the SD's Partial Euclidean Distance (PED) $d_{v}$ based on (19) and (24) is defined as (25), and the PED increment $\Delta_{v-1}$ is given by (26), where the coefficients $\left\{\widetilde{l}_{v-t+1,1}\right\}_{t=1}^{v}$ are elements in $\widetilde{\mathbf{L}}_{\mathbf{v}}$ obtained from $\widetilde{\mathbf{L}}_{\mathbf{v}} \widetilde{\mathbf{L}}_{\mathbf{v}}^{T}=\widetilde{\mathbf{C}}_{\mathbf{v}}^{-1}$. According to Proposition 1, we always have $\left\{l_{N_{w}-t+1, N_{w}-v+1}=\widetilde{l}_{v-t+1,1}\right\}_{t=1}^{v}$. The previous ringamplitudes $\left\{\Gamma_{t}\right\}_{t=1}^{v-1}$ in $\widetilde{\mathbf{C}}_{\mathbf{v}}$ of (21) are known from previous SD search, and hence there is a total of $M_{A}$ candidates for $\Gamma_{v}$, which determines $M_{A}$ candidates for $\widetilde{\mathbf{L}}_{\mathbf{v}}$. Moreover, the previous phases $\left\{\bar{\Omega}_{t}\right\}_{t=1}^{v-1}$ have also been decided, and hence there are $M_{P}$ candidates for $\omega_{v-1}$ in (26). The ring-amplitudedependent phase rotations $\left\{\bar{\Psi}_{t}\right\}_{t=1}^{v-1}$ and $\psi_{v-1}$ are explicitly determined by the ring-amplitudes $\left\{\Gamma_{t}\right\}_{t=1}^{v}$.

Furthermore, the determinant term in (26) is given by $\Xi_{v}=N_{R} \cdot \ln \left[\left(\Gamma_{v}^{2} \rho_{0}+N_{0}\right)-\widetilde{\mathbf{e}}_{v}^{T} \widetilde{\mathbf{C}}_{\mathbf{v}-\mathbf{1}}^{-1} \widetilde{\mathbf{e}}_{v}\right]-\xi_{v-1}$. We note that in order to retain the full MSDD capability, all the MSDSD's PED increment values of (26) have to be non-negative. For this reason, the extra constant of $\xi_{v-1}=\min _{\forall \Gamma_{1} \cdots \forall \Gamma_{v}} N_{R}$. $\ln \left[\left(\Gamma_{v}^{2} \rho_{0}+N_{0}\right)-\widetilde{\mathbf{e}}_{v}^{T} \widetilde{\mathbf{C}}_{\mathbf{v}-\mathbf{1}}^{-1} \widetilde{\mathbf{e}}_{v}\right]$ is introduced in the determinant term $\Xi$, which is similar to the case of adding $K_{i}$ for the soft-decision SD's PED in (23)-(25) of [36]. We note that adding a constant of $\left(\sum_{v=2}^{N_{w}}-\xi_{v-1}\right)$ to the MSDD metric of (18) does not impose any performance difference, and the constants $\left\{\xi_{v-1}\right\}_{v=2}^{N_{w}}$ are pre-evaluated and pre-stored in an off-line fashion, before performing MSDSD. In summary, the only variable in the determinant term $\Xi_{v}$ is $\Gamma_{v}$, and hence there are a total of $M_{A}$ candidates for $\Xi_{v}$.

\section{B. Schnorr-Euchner Search Strategy}

Based on the PED defined in (25), the MSDSD algorithm of [19] may be invoked, but its "sortDelta" subfunction formulated for the Schnorr-Euchner search strategy [34] should be revised as summarized in Table $\mathrm{I}^{2}$. Owing to the fact that the MSDD model of (10) stores the received samples in a reverse order compared to [19], the MSDSD algorithm should commence from index $v=1$, and the sphere radius is updated at index $v=N_{w}$. The SD search terminates, when the index of $v=2$ is reached again without finding any solution in the search sphere. The range for the child node counter $n_{v-1}$ in Table $\mathrm{I}$ is given by $0 \leq n_{v-1} \leq(M-1)$ throughout the SD search, which accords with the constellation point index range. Moreover, similar to the pseudocode presented in [19], the MSDSD may initialize the PED as $d_{1}=0$ for the sake of simplicity, but the $\Gamma_{1}$-related term $d_{1}=\left\|l_{N_{w}, N_{w}} \mathbf{Y}_{1}\right\|^{2}+N_{R} \cdot \ln \left(\Gamma_{1}^{2} \rho_{0}+N_{0}\right)$ should be added to the SD's output radius before comparing EDs over $\Gamma_{1}$ in (18).

It is worth noting that all the candidates of $\widetilde{\mathbf{L}}_{\mathbf{v}}$ and $\Xi_{v}$ over $\left\{\Gamma_{t}\right\}_{t=1}^{v}$ seen in (26) are pre-evaluated and pre-stored in an offline fashion. They remain fixed as long as the constellation as well as $N_{w}, N_{0}$ and $f_{d}$ are fixed. There is a total of $\sum_{v=1}^{N_{w}} M_{A}^{v}$ candidates for $\widetilde{\mathbf{L}}_{\mathbf{v}}$ and $\Xi_{v}$ stored in memory. As a special case of DAPSK associated with $M_{A}=1$, the DPSK only has to evaluate and store a single candidate for the constant $\widetilde{\mathbf{L}}_{\mathbf{N}_{\mathbf{w}}}=\mathbf{L}$.

We also note that the SD tree-search strategies include both K-Best and depth-first. Moreover the SD constellation-search

\footnotetext{
${ }^{2}$ We note that the subscript $m \in\{0, \cdots, M-1\}$ represents the datacarrying Gray coded constellation point index which may be directly translated back to binary source bits as $\left[b_{1} \cdots b_{\mathrm{BPS}}\right]=\operatorname{dec} 2 \operatorname{bin}(m)$. Furthermore, the subscript $\bar{m} \in\{\overline{0}, \cdots, \overline{M-1}\}$ represents the constellation point index ordered according to the increasing values of PED increment $\Delta_{v-1}$.
} 


$$
\begin{gathered}
d_{v}=\left\|l_{N_{w}, N_{w}} \mathbf{Y}_{1}\right\|^{2}+N_{R} \cdot \ln \left(\Gamma_{1}^{2} \rho_{0}+N_{0}\right)+\sum_{\bar{v}=2}^{v}\left\|\sum_{t=1}^{\bar{v}} l_{N_{w}-t+1, N_{w}-\bar{v}+1} \bar{\Psi}_{t}^{*} \bar{\Omega}_{t}^{*} \mathbf{Y}_{t}\right\|^{2} \\
+N_{R} \cdot \sum_{\bar{v}=2}^{v} \ln \left[\left(\Gamma_{\bar{v}}^{2} \rho_{0}+N_{0}\right)-\widetilde{\mathbf{e}}_{\bar{v}}^{T} \widetilde{\mathbf{C}}_{\overline{\mathbf{v}}-1}^{-1} \widetilde{\mathbf{e}}_{\bar{v}}\right]=d_{v-1}+\Delta_{v-1} . \\
\Delta_{v-1}=\left\|\widetilde{l}_{1,1} \bar{\Psi}_{v-1}^{*} \bar{\Omega}_{v-1}^{*} \mathbf{Y}_{v}+\omega_{v-1} \psi_{v-1}\left(\sum_{t=1}^{v-1} \widetilde{l}_{v-t+1,1} \bar{\Psi}_{t}^{*} \bar{\Omega}_{t}^{*} \mathbf{Y}_{t}\right)\right\|^{2}+\Xi_{v},
\end{gathered}
$$

\begin{tabular}{|ll|}
\hline Subfunction: $\quad\left[\left\{\Delta_{v}^{\bar{m}}\right\}_{\bar{m}=0}^{M-1},\left\{x_{v-1}^{\bar{m}}\right\}_{\bar{m}=0}^{M-1}, n_{v-1}\right]=\operatorname{sortDelta}\left(\left\{\mathbf{Y}_{t}\right\}_{t=1}^{v},\left\{\hat{\Gamma}_{t}\right\}_{t=1}^{v-1},\left\{\hat{\Psi}_{t}\right\}_{t=1}^{v-1},\left\{\hat{\Omega}_{t}\right\}_{t=1}^{v-1}\right)$ \\
Requirement: $\quad$ Both $\widetilde{\mathbf{L}}_{\mathbf{v}}$ and $\Xi\left(\left\{\hat{\Gamma}_{t}\right\}_{t=1}^{v-1}, \Gamma_{v}\right)=\Xi_{v}$ in (26) are pre-evaluated and pre-stored. & \\
\hline $1: \quad$ for $\mathrm{m}=0$ to $M-1$ & //visit all $M$ child nodes. \\
2: $\quad$ ADPSK/TADPSK/TADPSK ${ }^{\mathrm{JM}}: \Gamma_{v}=\gamma_{v-1}^{a} ;$ DAPSK/TDAPSK/TDAPSK ${ }^{\mathrm{JM}}: \Gamma_{v}=\gamma_{v-1}^{a} \cdot \hat{\Gamma}_{v-1}$ & //visit $x_{v-1}^{m}=\gamma_{v-1}^{a} \cdot \omega_{v-1}^{p} \cdot \psi_{v-1}^{a}$. \\
$3: \quad \Delta_{v-1}^{m}=\left\|\widetilde{l}_{1,1} \hat{\Psi}_{v-1}^{*} \hat{\Omega}_{v-1}^{*} \mathbf{Y}_{v}+\omega_{v-1}^{p} \psi_{v-1}^{a}\left(\sum_{t=1}^{v-1} \widetilde{l}_{v-t+1,1} \hat{\Psi}_{t}^{*} \hat{\Omega}_{t}^{*} \mathbf{Y}_{t}\right)\right\|^{2}+\Xi\left(\left\{\hat{\Gamma}_{t}\right\}_{t=1}^{v-1}, \Gamma_{v}\right)$ & //evaluate PED increments of $(26)$. \\
$4: \quad$ end for & //initialize child node counter. \\
$5: \quad n_{v-1}=0$ & //sort $\left\{\Delta_{v-1}^{m}\right\}_{m=0}^{M-1}$ in increasing order. \\
6: $\quad\left[\left\{\Delta_{v-1}^{\bar{m}}\right\}_{\bar{m}=0}^{M-1},\left\{x_{v-1}^{\bar{m}}\right\}_{\bar{m}=0}^{M-1}\right]=\operatorname{sort}\left(\left\{\Delta_{v-1}^{m}\right\}_{m=0}^{M-1}\right)$ & \\
\hline
\end{tabular}

TABLE I

PSEUdO-CODE FOR THE SCHNORR-EUCHNER SEARCH STRATEGY TAILORED FOR MSDSD AIDED DQAM.

strategies include both the Fincke-Pohst enumeration [37] and the Schnorr-Euchner search strategy [34]. In order to guarantee that the MSDSD achieves the same detection capability as the MSDD, the depth-first [38] and the Schnorr-Euchner strategy [34] constitute the default choice for the MSDSD algorithms [18], [19].

More explicitly, according to the depth-first tree-search strategy [38], the SD increases the index as $(v=v+1)$ or decreases the index as $(v=v-1)$, when the new PED value $d_{v}$ of (25) is lower or higher than the search radius, respectively. Moreover, according to the Schnorr-Euchner constellationsearch strategy [34], when the SD visits a specific index $v$ for the $(\bar{m}+1)$-th time, the constellation point considered is the one associated with the $(\bar{m}+1)$-th lowest PED increment value $\Delta_{v-1}$ of (26). Therefore, the practical approach to the implementation of the Schnorr-Euchner strategy [34] is to sort the $M$ PED increment values $\Delta_{v-1}$ in increasing order, as seen in Table I.

An example of the HDD-MSDSD aided 16-TADPSK ${ }^{\mathrm{JM}}(2,8)$ using the Schnorr-Euchner search strategy of Table I is portrayed in Fig. 2. Explicitly. Fig. 2 shows that when the SD visits $v=2$ and $v=3$ for the first time in Steps (1) and (2), the "sortDelta" subfunction sorts the $M=16$ values of $\Delta_{v-1}$ in increasing order, where the best candidate associated with the lowest $\Delta_{v-1}$ is chosen. The PED is updated as $d_{v}=d_{v-1}+\Delta_{v-1}$ in each step, and the search radius is updated to $d_{N_{w}}=5.42$ in Step (2). For Step (3), the SD decreases its index to $v=2$ in order to visit the secondbest candidate, whose PED value of $d_{2}=9.88$ turns out to be higher than the SD radius. Hence the SD search is terminated.

\section{Reduced-Complexity Schnorr-Euchner Search Strategy}

When DQAM ${ }^{\mathrm{JM}}$ is employed, the Schnorr-Euchner search strategy of Table I, which exhaustively visits all $M$ constellation points is the only choice. However, a reducedcomplexity search strategy may be conceived for detecting DQAM constellations including ADPSK, DAPSK, TADPSK and TDAPSK, which modulate the ring-amplitude and phase independently. In order to achieve this goal, we separate the PED increment of (26) into two terms as:

$$
\Delta_{v-1}=\Delta_{v-1}^{\Gamma}+\Delta_{v-1}^{\omega \mid \Gamma}
$$

where the ring-amplitude-related term is given by:

$$
\Delta_{v-1}^{\Gamma}=\left\|\widetilde{l}_{1,1} \bar{\Psi}_{v-1}^{*} \bar{\Omega}_{v-1}^{*} \mathbf{Y}_{v}\right\|^{2}+\left\|\sum_{t=1}^{v-1} \widetilde{l}_{v-t+1,1} \bar{\Psi}_{t}^{*} \bar{\Omega}_{t}^{*} \mathbf{Y}_{t}\right\|^{2}+\Xi_{v},
$$

and $\Delta_{v-1}^{\Gamma}$ only has $M_{A}$ candidates over the single variable $\Gamma_{v}$. We note that $\Delta_{v-1}^{\Gamma}$ of (28) is invariant over the different phase candidates $\omega_{v-1}$ in (27). Moreover, the phase-related term conditioned on the ring-amplitude $\Delta_{v-1}^{\omega \mid \Gamma}$ in (27) is given by:

$$
\begin{aligned}
\Delta_{v-1}^{\omega \mid \Gamma} & =2 \Re\left[\omega_{v-1}^{*} \widetilde{l}_{1,1} \psi_{v-1}^{*} \bar{\Psi}_{v-1}^{*} \bar{\Omega}_{v-1}^{*} \mathbf{Y}_{v}\left(\sum_{t=1}^{v-1} \widetilde{l}_{v-t+1,1} \bar{\Psi}_{t}^{*} \bar{\Omega}_{t}^{*} \mathbf{Y}_{t}\right)^{H}\right] \\
& =-2 \Re\left(\omega_{v-1}^{*} z_{v-1}^{\omega \mid \Gamma}\right),
\end{aligned}
$$

where the decision variable is formulated as:

$$
z_{v-1}^{\omega \mid \Gamma}=\mathbf{Y}_{v-1}^{\mathrm{SD}}\left(\mathbf{H}_{v-1}^{\mathrm{SD}}\right)^{H} .
$$

The $N_{R}$-element row vectors $\mathbf{Y}_{v-1}^{\mathrm{SD}}=\widetilde{l}_{1,1} \psi_{v-1}^{*} \bar{\Psi}_{v-1}^{*} \bar{\Omega}_{v-1}^{*} \mathbf{Y}_{v}$ and $\mathbf{H}_{v-1}^{\mathrm{SD}}=-\sum_{t=1}^{v-1} \widetilde{l}_{v-t+1,1} \bar{\Psi}_{t}^{*} \bar{\Omega}_{t}^{*} \mathbf{Y}_{t}$ seen in (30) may be interpreted as the equivalent "received signal vector" and "fading vector" formulated for detecting $\omega_{v-1}$ in (29). More explicitly, when the ring-amplitude $\Gamma_{v}$ is assumed to be fixed, both $\left\{\widetilde{l}_{v-t+1,1}\right\}_{t=1}^{v}$ and $\psi_{v-1}$ of (29) are given, hence there are $M_{P}$ candidates for $\Delta_{v-1}^{\omega \mid \Gamma}$ over the single variable $\omega_{v-1}$. Therefore, given a specific $\Gamma_{v}$, finding the local minimum $\Delta_{v-1}^{\omega \mid \Gamma}$ of (29) over all the $M_{P}$ phase candidates of $\omega_{v-1}$ is equivalent to minimizing $\left|z_{v-1}^{\omega \mid \Gamma}-\omega_{v-1}\right|^{2}=\left|z_{v-1}^{\omega \mid \Gamma}\right|^{2}+1-2 \Re\left(\omega_{v-1}^{*} z_{v-1}^{\omega \mid \Gamma}\right)$, where $\left|z_{v-1}^{\omega \mid \Gamma}\right|^{2}+1$ is a constant. As a result, the decision variable $z_{v-1}^{\omega \mid \Gamma}$ of (30) may be directly used for detecting the phase variable $\omega_{v-1}$.

More explicitly, the locally optimum phase associated with a specific ring-amplitude $\Gamma_{v}$ may be directly given by $\omega_{v-1}=$ $\exp \left(j \frac{2 \pi}{M_{P}} \check{p}\right)$, where $\check{p}=\left\lfloor\frac{M_{P}}{2 \pi} \angle z_{v-1}^{\omega \mid \Gamma}\right\rceil$, and the remaining local phase candidates may be visited later in a zigzag fashion by the SD in the same way as the MSDSD aided DPSK [18]. Once the local phase candidates related to each $\Gamma_{v}$ have been determined, the globally minimum PED increment is found by comparing the $M_{A}$ local candidates for $\Delta_{v-1}$ of (27) over the variable $\Gamma_{v}$. Based on this design, the MSDSD algorithm of [18] may be invoked for DQAM, which modulates the ring-amplitude and phase separately, but the "findBest" and 


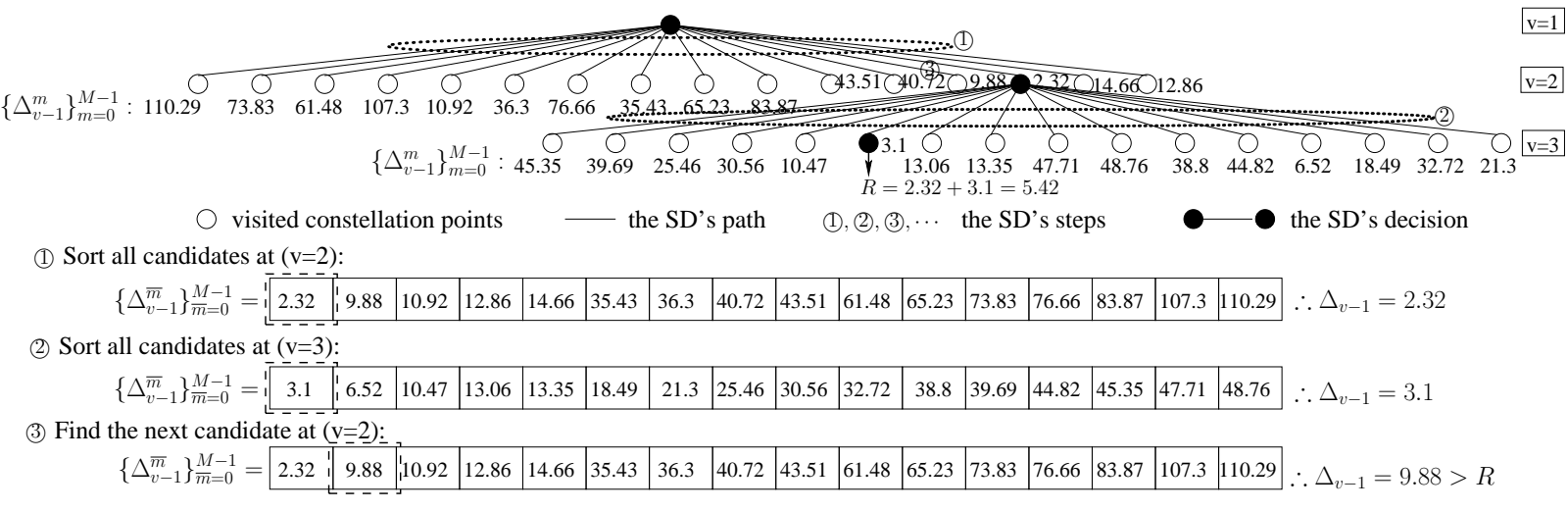

Fig. 2. Example of HDD-MSDSD aided 16-TADPSK ${ }^{\mathrm{JM}}(2,8)$ recorded at $\mathrm{SNR}=15 \mathrm{~dB}$, where we have $N_{R}=2$ and $N_{w}=3$.

"findNext" subfunctions of [18] should be replaced by those summarized in Table II.

For further illustration, the HDD-MSDSD aided 16$\operatorname{ADPSK}(2,8)$ is exemplified in Fig. 3, which invokes the same depth-first tree-search strategy as the example of HDDMSDSD aided 16-TDAPSK ${ }^{\mathrm{JM}}(2,8)$ of Fig. 2. The difference is that the Schnorr-Euchner constellation-search strategy is now simplified. In more detail, Fig. 3 shows that when the SD visits $v=2$ for the first time in Step (1), the "findBest" subfunction of Table II firstly evaluates the decision variables $\left\{z_{v-1}^{\omega \mid \Gamma^{a}}\right\}_{a=0}^{1}$ of (30) for the $M_{A}=2$ ring-amplitudes, and then their phase indices are directly given by $\left\{\check{p}=\left\lfloor\frac{M_{P}}{2 \pi} \angle z_{v-1}^{\omega \mid \Gamma^{a}}\right\rceil\right\}_{a=0}^{1}$. The PED increment values for these two local candidates of ( $a=$ $0, \check{p}=3)$ and $(a=1, \check{p}=3)$ are evaluated according to (27), and then $(a=0, \check{p}=3)$ is chosen for $v=2$, which has the global minimum of $\Delta_{v-1}=\min \left\{\Delta_{v-1}^{4}, \Delta_{v-1}^{5}\right\}=3.14$. Then the SD increases its index to $v=3$ in Step (2), where again, the "findBest" subfunction of Table II is invoked. The SD radius is updated to $d_{N_{w}}=4.7$ in Step (2). Moreover, when the SD index is decremented back to $v=2$ in Step (3), the "findNext" subfunction of Table II firstly updates a new local phase candidate for $a=0$, because $(a=0, \check{p}=3)$ was previously chosen for $v=2$, and then the next global candidate for $v=2$ is found by comparing $\Delta_{v-1}=\min \left\{\Delta_{v-1}^{8}, \Delta_{v-1}^{5}\right\}=5.65$, which is higher than the SD radius, as seen in Fig. 3. Hence the SD search is terminated.

In summary, given the same number of SD steps, the HDDMSDSD aided 16-DAPSK $(2,8)$ of Fig. 3 visits a considerably lower number of constellation points than the HDD-MSDSD aided 16-TDAPSK ${ }^{\mathrm{JM}}(2,8)$ of Fig. 2.

\section{Decision-Feedback Differential Detection}

It was demonstrated in [12], [13] that the DFDD aided DQAM is capable of estimating the current CSI sample based on the previous decisions, so that coherent detection may be performed. However, the aforementioned contributions ignored the problem of having a ring-amplitude-dependent channel correlation matrix. With the advent of solving this problem for MSDSD, in this section, we propose to further improve the conventional DFDD solution of [12], [13], so that the DFDD aided DQAM becomes equivalent to the optimum MSDD aided DQAM of Sec. III operating in decision-feedback mode.
Firstly, the most recent received signal vector within an observation window is given by:

$$
\mathbf{Y}_{N_{w}}=s_{N_{w}} \mathbf{H}_{N_{w}}+\mathbf{V}_{N_{w}} \approx \Omega_{N_{w}} \Psi_{N_{w}} \mathbf{H}_{N_{w}}^{\mathrm{ref}}+\mathbf{V}_{N_{w}} .
$$

The reference $\mathbf{H}_{N_{w}}^{\mathrm{ref}}$ in (31) is output from a linear prediction filter as [13]:

$$
\mathbf{H}_{N_{w}}^{\mathrm{ref}}=\sum_{v=1}^{N_{w}-1} \bar{w}_{v} \mathbf{Y}_{v} /\left(\hat{\Omega}_{v} \hat{\Psi}_{v}\right)=\overline{\mathbf{w}}^{T}\left(\hat{\mathbf{O}}^{\bar{N}_{w}}\right)^{H}\left(\hat{\mathbf{P}}^{\bar{N}_{w}}\right)^{H} \mathbf{Y}^{\bar{N}_{w}},
$$

where $\overline{\mathbf{w}}=\left[\bar{w}_{N_{w}-1}, \cdots, \bar{w}_{1}\right]^{T}$ represents the filter taps, while the diagonal matrices $\hat{\mathbf{P}}^{\bar{N}_{w}}$ and $\hat{\mathbf{O}}^{N_{w}}$ are given by the previous decisions on $\mathbf{P}$ and $\mathbf{O}$ of (10) eliminating $\Omega_{N_{w}}$ and $\Psi_{N_{w}}$, respectively. Moreover, $\mathbf{Y}^{\bar{N}_{w}}$ in (32) is given by $\mathbf{Y}$ of (10) eliminating $\mathbf{Y}_{N_{w}}$. The DFDD aims for minimizing the Mean Square Error (MSE):

$$
\begin{aligned}
\sigma_{\mathrm{MSE}}^{2} & =E\left\{\left\|\mathbf{Y}_{N_{w}} /\left(\Omega_{N_{w}} \Psi_{N_{w}}\right)-\mathbf{H}_{N_{w}}^{\mathrm{ref}}\right\|^{2}\right\} \\
= & \Gamma_{N_{w}}^{2}+N_{0}-2 E\left\{\Gamma_{N_{w}} \mathbf{H}_{N_{w}}\left(\mathbf{Y}^{\bar{N}_{w}}\right)^{H} \hat{\mathbf{P}}^{\bar{N}_{w}} \hat{\mathbf{O}}^{\bar{N}_{w}}\right\} \overline{\mathbf{w}} \\
& +\overline{\mathbf{w}}^{T} E\left\{\left(\hat{\mathbf{O}}^{\bar{N}_{w}}\right)^{H}\left(\hat{\mathbf{P}}^{\bar{N}_{w}}\right)^{H} \mathbf{Y}^{\bar{N}_{w}}\left(\mathbf{Y}^{\bar{N}_{w}}\right)^{H} \hat{\mathbf{P}}^{\bar{N}_{w}} \hat{\mathbf{O}}^{\bar{N}_{w}}\right\} \overline{\mathbf{w}} \\
= & \Gamma_{N_{w}}^{2}+N_{0}-2 \widetilde{\mathbf{e}}_{N_{w}}^{T} \overline{\mathbf{w}}+\overline{\mathbf{w}}^{T} \widetilde{\mathbf{C}}_{\mathbf{N}_{\mathbf{w}}-\mathbf{1}} \overline{\mathbf{w}}
\end{aligned}
$$

where the auto-correlation $\widetilde{\mathbf{C}}_{\mathbf{N}_{\mathrm{w}}-1}$ and cross-correlation $\widetilde{\mathbf{e}}_{N_{w}}$ are given by the submatrices of $\widetilde{\mathbf{C}}_{\mathbf{v}}$ in (21) associated with $v=N_{w}$. Therefore, the MMSE solution for $\frac{\partial \sigma_{\mathrm{MSE}}^{2}}{\partial \overline{\mathbf{w}}}=0$ is given by [13]:

$$
\overline{\mathbf{w}}=\widetilde{\mathbf{C}}_{\mathbf{N}_{\mathbf{w}}-\mathbf{1}}^{-1} \widetilde{\mathbf{e}}_{N_{w}} .
$$

As a result, the MSE of (33) is now simply given by:

$$
\sigma_{\mathrm{MSE}}^{2}=\Gamma_{N_{w}}^{2}+N_{0}-\widetilde{\mathbf{e}}_{N_{w}}^{T} \widetilde{\mathbf{C}}_{\mathbf{N}_{\mathbf{w}}-\mathbf{1}}^{-1} \widetilde{\mathbf{e}}_{N_{w}} .
$$

The DFDD opts for maximizing the a posteriori probability of $p\left(\Gamma_{N_{w}}, \Omega_{N_{w}} \mid \mathbf{Y}_{N_{w}}\right)$, which is equivalent to the following conditional probability when $\gamma_{N_{w}-1}$ and $\omega_{N_{w}-1}$ are both equiprobable:

$p\left(\mathbf{Y}_{N_{w}} \mid \Gamma_{N_{w}}, \Omega_{N_{w}}\right)=\frac{1}{\pi \sigma_{M S E}^{2}} \exp \left(-\frac{\left\|\mathbf{Y}_{N_{w}}-\Omega_{N_{w}} \Psi_{N_{w}} \mathbf{H}_{N_{w}}^{\mathrm{ref}}\right\|^{2}}{\sigma_{M S E}^{2}}\right)$.

Equivalently, the DFDD minimizes the decision metric of (37), where we have $\widetilde{\Xi}_{N_{w}}=\ln \left(\sigma_{M S E}^{2}\right)=$ $\ln \left[\left(\Gamma_{N_{w}}^{2}+N_{0}\right)-\widetilde{\mathbf{e}}_{N_{w}}^{T} \widetilde{\mathbf{C}}_{\mathbf{N}_{\mathbf{w}}-\mathbf{1}}^{-1} \widetilde{\mathbf{e}}_{N_{w}}\right] \quad$ according to 


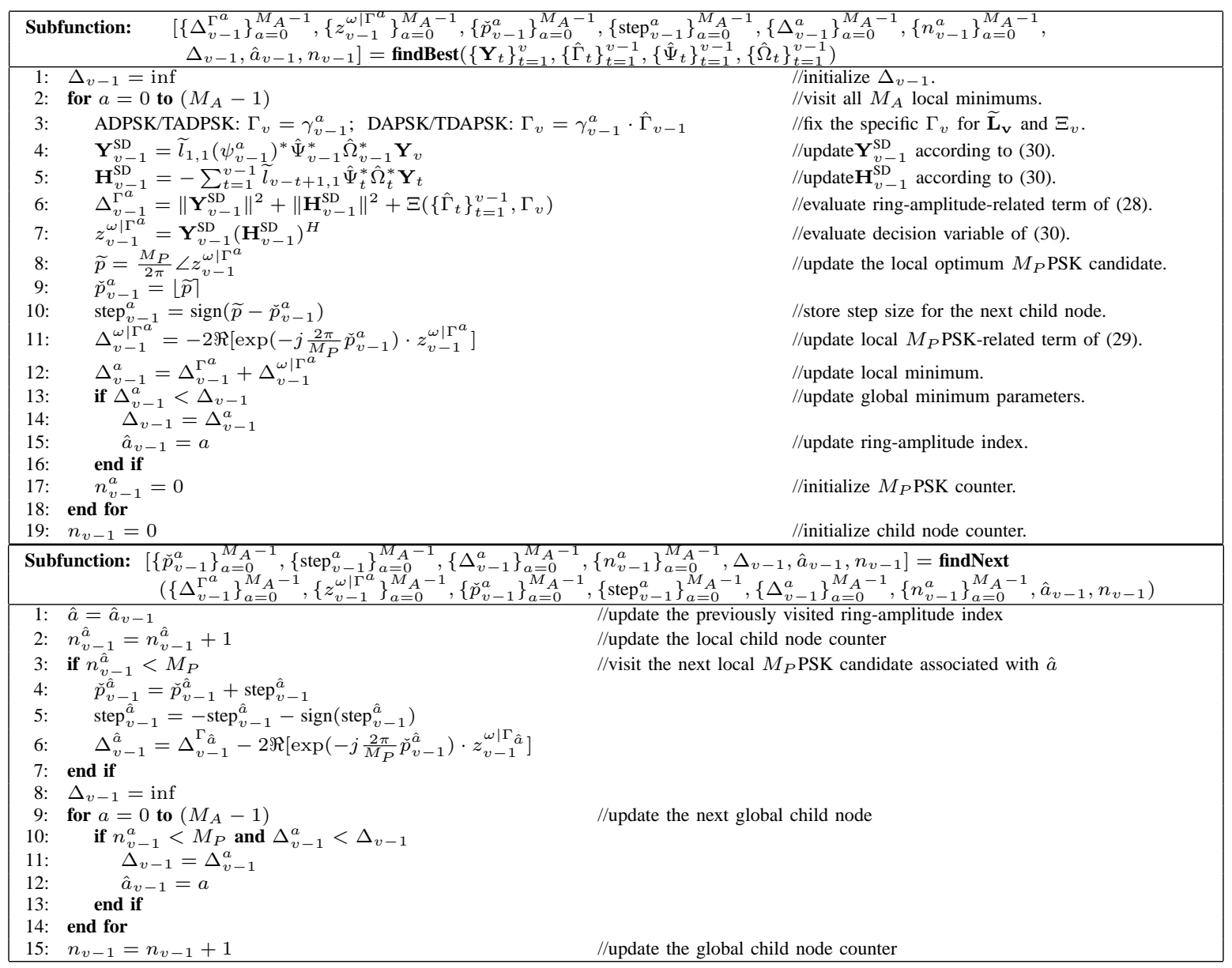

TABLE II

PSEUDO-CODE FOR THE REDUCED-COMPLEXITY SCHNORR-EUCHNER SEARCH STRATEGY TAILORED FOR MSDSD AIDED DQAM, WHICH MODULATE THE RING-AMPLITUDE AND PHASE SEPARATELY.

(35). Furthermore, according to (23), we have $\widetilde{Q}_{N_{w}-1}=\left(\Gamma_{N_{w}}^{2} \rho_{0}+N_{0}-\widetilde{\mathbf{e}}_{N_{w}}^{T} \widetilde{\mathbf{C}}_{\mathbf{N}_{\mathbf{w}}-\mathbf{1}}^{-1} \widetilde{\mathbf{e}}_{N_{w}}\right)^{-1}=1 / \sigma_{M S E}^{2}$ and we also have $\widetilde{Q}_{N_{w}-1}=l_{1,1}^{2}$ as a benefit of the relationship of $\mathbf{C}^{-1}=\mathbf{L} \mathbf{L}^{T}$. Therefore, the MSE of (35) may be rewritten as $\sigma_{M S E}^{2}=1 / \widetilde{Q}_{N_{w}-1}=1 / l_{1,1}^{2}$, which results in the DFDD decision metric presented in (37). Furthermore, we have $\widetilde{\mathbf{D}}_{\mathbf{N}_{\mathbf{w}}-\mathbf{1}}=\widetilde{\mathbf{e}}_{N_{w}}$ according to (22), which results in $-l_{1,1} \bar{w}_{t}=l_{N_{w}-t+1,1}$ because $-\widetilde{Q}_{N_{w}-1} \widetilde{\mathbf{C}}_{\mathbf{N}_{\mathbf{w}}-\mathbf{1}}^{-1} \widetilde{\mathbf{e}}_{N_{w}}$ is in the first column of $\mathbf{C}^{-1}$ according to (23) and also because of the relationship of $\mathbf{C}^{-1}=\mathbf{L L}^{T}$. As a result, the DFDD metric of (37) is completely equivalent to the MSDSD's PED increment of (26) associated with $v=N_{w}$. Therefore, the DFDD aided DQAM ${ }^{\mathrm{JM}}$ may be simply completed by the "sortDelta" in Table I, and the DFDD aided DQAM which separately modulates the ring-amplitude and phase may be implemented by the "findBest" of Table II, where both subfunctions are supposed to be associated with $v=N_{w}$.

\section{Vi. Performance Results}

In this section, we offer simulation results of capacity, BER and complexity for the MSDSD and DFDD aided DQAM. Without loss of generality, we focus our attention on the 16level and 64-level DQAM constellations introduced in Sec. II, where the default Star QAM configurations are $\left(M_{A}, M_{P}\right)=$ $(2,8)$ for $M=16$ as seen in [1], [2], [4]-[6], [10]-[13], [24],
[28], [29], [31], [32], [39], [40] and $\left(M_{A}, M_{P}\right)=(4,16)$ for $M=64$ as demonstrated in [4], [7], [30]-[32].

\section{A. Capacity Comparison}

Let us firstly determine the Discrete-input Continuousoutput Memoryless Channel (DCMC) capacity [20] of the MSDD aided DQAM systems. For the differential-amplitude DQAM constellations of DAPSK and TDAPSK, the first transmitted ring-amplitude is treated as an equiprobable variable, i.e. we have $\left\{p\left(\Gamma^{\widetilde{a}}\right)=p\left(\Gamma^{\bar{a}}\right)=\frac{1}{M_{A}}\right\}_{\forall \widetilde{a}, \forall \bar{a}}$. Therefore, the DCMC capacity of MSDD aided differential-amplitude DQAM is given by (38), where $p\left(\mathbf{Y} \mid \overline{\mathbf{S}}^{i}, \Gamma^{a}\right)$ is given by (13), while the conditions of $\overline{\mathbf{S}}=\overline{\mathbf{S}}^{i}$ and $\Gamma_{1}=\Gamma^{a}$ indicate that $\mathbf{Y}$ is obtained by transmitting $\overline{\mathbf{S}}^{i}$ and $\Gamma^{a}$. By contrast, the absoluteamplitude DQAM constellations of ADPSK and TADPSK can only employ HDD-MSDD, which implies that the full DCMC capacity is achieved, when the a priori information representing $\Gamma_{1}$ is available from decision feedback, i.e. when we have $\left\{p\left(\Gamma^{\widetilde{a}}\right)=p\left(\Gamma^{\bar{a}}\right)=1\right\}_{\widetilde{a}=\bar{a}=a}$ and $\left\{p\left(\Gamma^{\widetilde{a}}\right)=p\left(\Gamma^{\bar{a}}\right)=\right.$

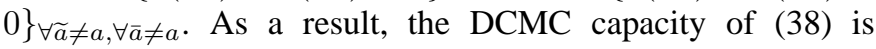
revised for HDD-MSDD aided absolute-amplitude DQAM as in (39).

The DCMC capacities of ADPSK and DAPSK formulated in (39) and (38) are portrayed in Fig. 4. First of all, Fig. 4(a) demonstrates that the CDD facilitated by MSDD associated 


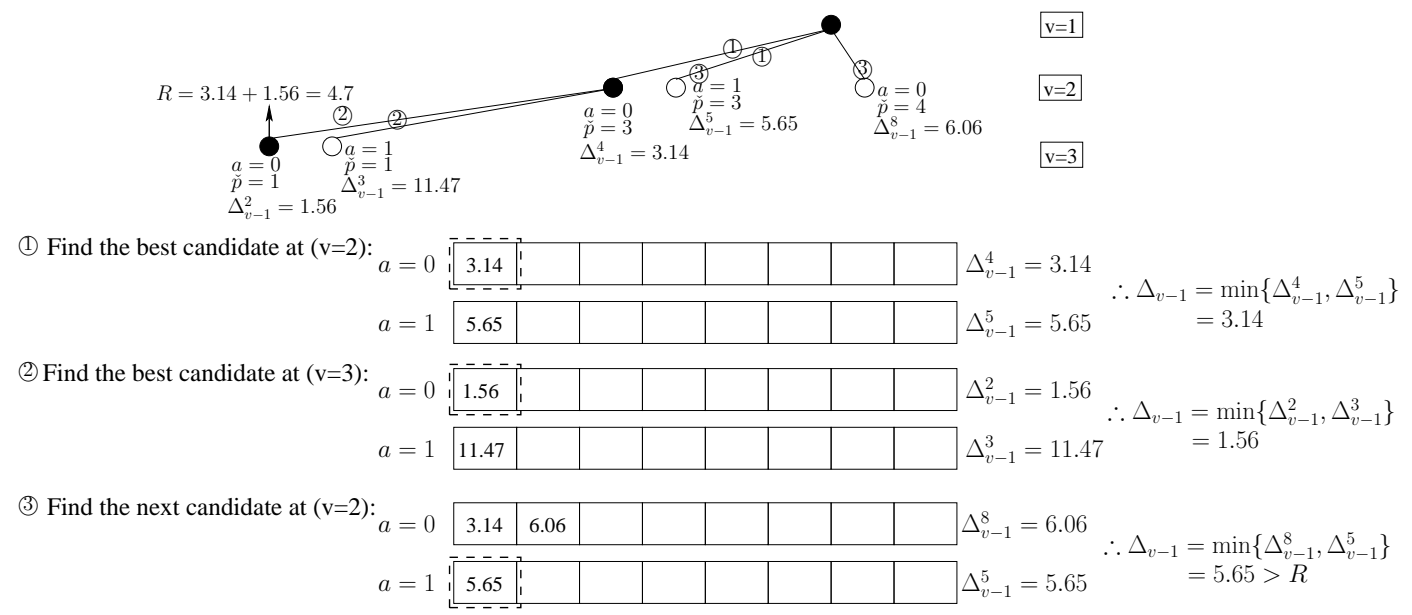

Fig. 3. Example of reduced-complexity HDD-MSDSD aided 16-ADPSK $(2,8)$ recorded at $\mathrm{SNR}=15 \mathrm{~dB}$, where we have $N_{R}=2$ and $N_{w}=3$.

$$
\begin{gathered}
d=\left\|l_{1,1} \hat{\Omega}_{N_{w}-1}^{*} \hat{\Psi}_{N_{w}-1}^{*} \mathbf{Y}_{N_{w}}-\omega_{N_{w}-1} \psi_{N_{w}-1}\left(\sum_{t=1}^{N_{w}-1} l_{1,1} \bar{w}_{t} \hat{\Psi}_{t}^{*} \hat{\Omega}_{t}^{*} \mathbf{Y} t\right)\right\|^{2}+\widetilde{\Xi}_{N_{w}} \\
C_{D C M C}^{M S D D}=\frac{\sum_{a=0}^{M_{A}-1} \sum_{i=0}^{M^{\left(N_{w}-1\right)}-1} E\left\{\log _{2}\left[\frac{M^{\left(N_{w}-1\right)} \cdot \sum_{\tilde{a}=0}^{M_{A}-1} p\left(\mathbf{Y} \mid \overline{\mathbf{S}}^{i}, \Gamma^{\tilde{a}}\right)}{\sum_{\bar{a}=0}^{M_{A}-1} \sum_{\bar{i}=0}^{M^{(N-1)}-1} p\left(\mathbf{Y} \mid \overline{\mathbf{S}}^{\bar{i}}, \Gamma^{\bar{a}}\right)}\right] \mid \overline{\mathbf{S}}=\overline{\mathbf{S}}^{i}, \Gamma_{1}=\Gamma^{a}\right\}}{\left(N_{w}-1\right) M_{A} M^{\left(N_{w}-1\right)}} . \\
C_{D C M C}^{H D D-M S D D}=\frac{\sum_{a=0}^{M_{A}-1} \sum_{i=0}^{M^{\left(N_{w}-1\right)}-1} E\left\{\log _{2}\left[\frac{M^{\left(N_{w}-1\right)} p\left(\mathbf{Y} \mid \overline{\mathbf{S}}^{i}, \Gamma^{a}\right)}{\sum_{\bar{i}=0}^{M^{\left(N_{w}-1\right)}-1} p\left(\mathbf{Y} \mid \overline{\mathbf{S}}^{\bar{i}}, \Gamma^{a}\right)}\right] \mid \overline{\mathbf{S}}=\overline{\mathbf{S}}^{i}, \Gamma_{1}=\Gamma^{a}\right\}}{\left(N_{w}-1\right) M_{A} M^{\left(N_{w}-1\right)}} .
\end{gathered}
$$

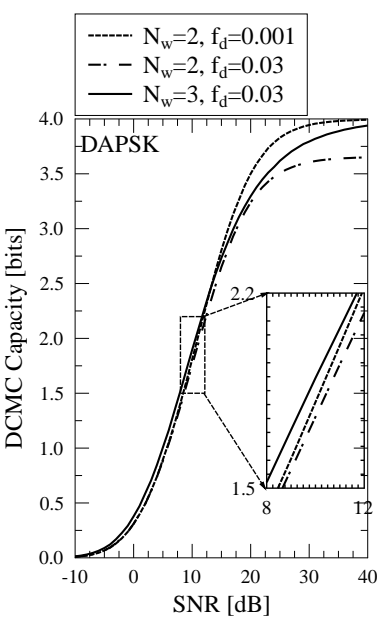

(a)

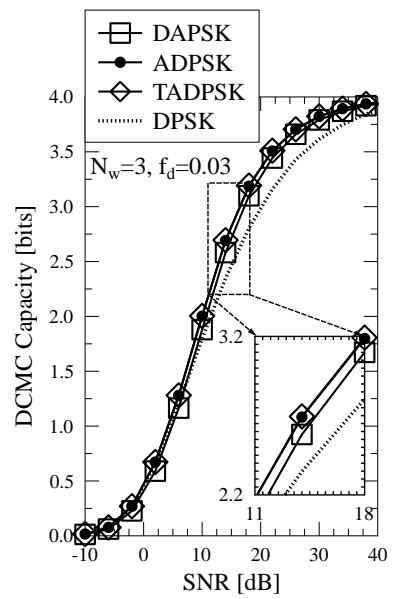

(b)

Fig. 4. Capacity of MSDD aided DQAM, where we have $N_{R}=$ 1. (a) MSDD aided 16-DAPSK(2,8). (b) MSDD/HDD-MSDD aided 16DAPSK $(2,8), 16-\operatorname{ADPSK}(2,8), 16-\operatorname{TADPSK}(2,8)$ and 16-DPSK, $N_{w}=3$, $f_{d}=0.03$.

\begin{tabular}{|l|c|c|}
\hline & $\min \left|x^{m}-x^{m^{\prime}}\right|$ & $\mathrm{E}\left(\left|x^{m}-x^{m^{\prime}}\right|\right)$ \\
\hline 16DAPSK(2,8) & 0.382683 & 1.60486 \\
\hline 16ADPSK(2,8) & 0.484061 & 1.35334 \\
\hline 16TADPSK(2,8) & 0.484061 & 1.35346 \\
\hline
\end{tabular}

TABLE III

COMPARISON OF DAPSK, ADPSK AND TADPSK CONSTELLATION DISTANCES.

with $N_{w}=2$ is unable to achieve the full DCMC capacity of $C_{\max }^{D C M C}=$ BPS even at high SNRs, when we have $f_{d}=0.03$. This capacity gap predicts an error floor for the CDD in rapidly fluctuating fading channels. Nonetheless, it is evidenced by Fig. 4(a) that the MSDD effectively mitigates the CDD's capacity gap in high SNR region and also improve the CDD's

performance in low SNR region, when $N_{w}$ is increased to 3 .

Secondly, the DCMC capacities of the DQAM constellations are further compared in Fig. 4(b), where the DPSK capacity is also portrayed as a benchmark. It is evidenced by Fig. 4(b) that the 16-DQAM constellations generally have a higher DCMC capacity than 16-DPSK, which verifies the claim that DQAM is more bandwidth efficient. Moreover, it is also demonstrated by Fig. 4(b) that both 16- $\operatorname{ADPSK}(2,8)$ and 16-DAPSK $(2,8)$ achieve a similar performance at high SNRs in uncoded systems, but HDD-MSDD aided 16-ADPSK $(2,8)$ may outperform MSDD aided 16-DAPSK $(2,8)$ in turbo detection aided coded systems operating at low SNRs. This feature is also observed in Table III, where the 16- $\operatorname{ADPSK}(2,8)$ constellation exhibits a higher minimum distance than 16$\operatorname{DAPSK}(2,8)$, which indicates a better performance for 16$\operatorname{ADPSK}(2,8)$ at low SNRs. Moreover, Fig. 4(b) shows furthermore that TADPSK does not achieve any noticeable capacity improvement over ADPSK. This is because although the twisted modulation [2], [13] increases the distance between the constellation points located on the different ring-amplitudes, the minimum distance that is determined by the adjacent constellation points located on the smallest amplitude ring remain unchanged, as confirmed by Table III. Nonetheless, since the ring-amplitude-dependent phase rotation imposes a correlation between the ring-amplitude and phase, the softdecision detection of the twisted modulation constellations is expected to be able to benefit from an improved iteration gain in turbo detection assisted coded systems, which will be further discussed in Sec. VIII.

As a closely related result, our simulations based on (38) and (39) also confirm that $\left(M_{A}, M_{P}\right)=(2,8)$ achieves higher 


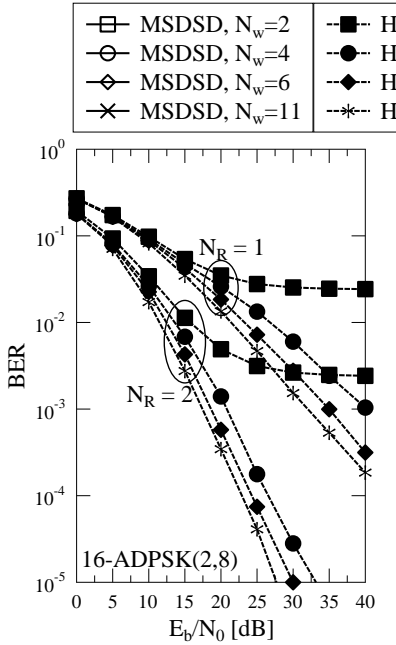

(a)
D-MSDSD, $\mathrm{N}_{\mathrm{w}}=2$
DD-MSDSD, $\mathrm{N}_{\mathrm{w}}=4$
D-MSDSD, $\mathrm{N}_{\mathrm{w}}=6$
D-MSDSD, $\mathrm{N}_{\mathrm{w}}=11$

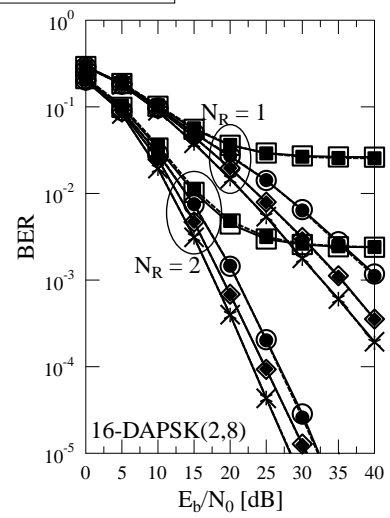

(b)

Fig. 5. BER performance of $\operatorname{HDD}-M \operatorname{MSDD}$ aided 16-ADPSK $(2,8)$ as well as MSDSD and HDD-MSDSD aided 16-DAPSK(2,8), where we have $f_{d}=$ 0.03. (a) 16-ADPSK $(2,8)$. (b) 16-DAPSK $(2,8)$.

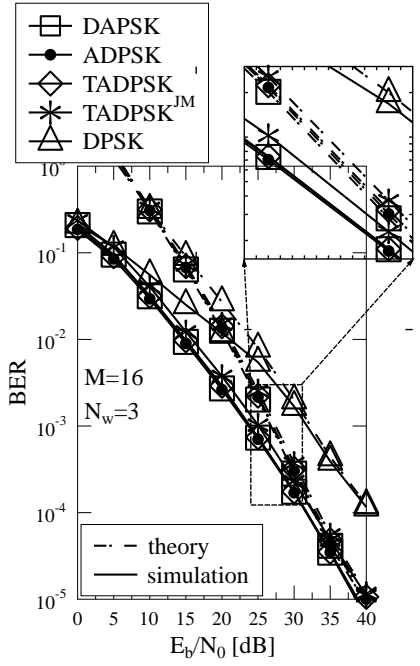

(a)

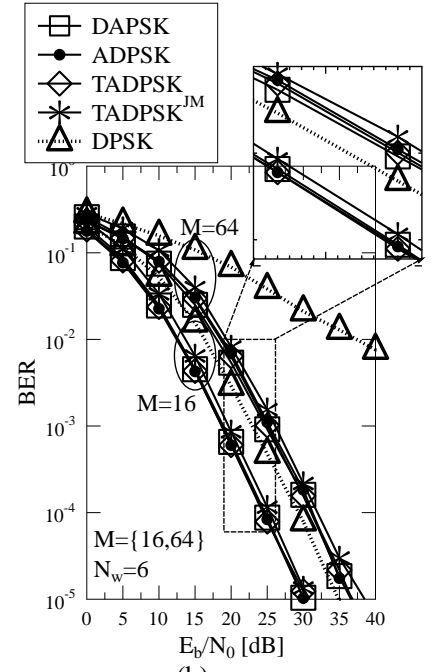

(b)

Fig. 6. BER performance comparison between DAPSK, ADPSK, TADPSK and TADPSK ${ }^{\mathrm{JM}}$ employing HDD-MSDSD, where we have $N_{R}=2$ and $f_{d}=0.03$. The performance result for MSDSD aided DPSK [18] is also portrayed as a benchmark. (a) $M=16, N_{w}=3$. (b) $M=\{16,64\}$, $N_{w}=6$.

capacities than $\left(M_{A}, M_{P}\right)=(4,4)$ for both 16-ADPSK and 16-DAPSK, albeit these curves are not included in this paper due to the associated space limit. It is worth noting that similar results can be found in [29], where $\left(M_{A}, M_{P}\right)=(2,8)$ also achieved a higher capacity than $\left(M_{A}, M_{P}\right)=(4,4)$ for 16DAPSK employing CDD.

\section{B. BER Performance Comparison}

The capacity results of Fig. 4 are further verified by the BER results of Fig. 5, which demonstrates that both HDD-MSDSD aided ADPSK and MSDSD aided DAPSK mitigate the error floor of the CDD encountered in rapidly fluctuating fading channels. Moreover, it is also demonstrated by Fig. 5(b) that HDD-MSDSD does not impose any significant performance loss on MSDSD for DAPSK. Therefore, the HDD-MSDSD is capable of facilitating both DAPSK and ADPSK detection in uncoded systems.

Fig. 6 further compares the BERs of DQAM constellations employing HDD-MSDSD, where the performance of MSDSD

aided DPSK [18] is also portrayed as a benchmark. The theoretical BER portrayed in Fig. 6(a) is evaluated according to:

$$
\bar{P}_{e} \approx \sum_{i=0}^{M^{\left(N_{w}-1\right)}} \sum_{\bar{i}=0, \bar{i} \neq i}^{M^{\left(N_{w}-1\right)}} \frac{d_{H}(i, \bar{i})}{M^{\left(N_{w}-1\right)}\left(N_{w}-1\right) \mathrm{BPS}} p\left(\overline{\mathbf{S}}^{i} \rightarrow \overline{\mathbf{S}}^{\bar{i}}\right)
$$

where $d_{H}(i, \bar{i})$ refers to the Hamming distance between the bit-mappings of $\overline{\mathbf{S}}^{i}$ and $\overline{\mathbf{S}}^{i}$, which is directly obtained by converting the indices $i$ and $\bar{i}$ back to $\left(N_{w}-1\right)$ BPS bits. Moreover, the Pairwise Error Probability (PEP) in (40) is evaluated according to $p\left(\overline{\mathbf{S}}^{i} \rightarrow \overline{\mathbf{S}}^{\bar{i}}\right)=p(D<0) \leq$ $\sum_{\mathrm{RH} \text { poles }} \operatorname{Res}\left(-\frac{\Phi_{D}(s)}{s}\right)$, which takes into account the residues at the poles located in the right-hand complex s plane [17]. More explicitly, the MSDD decision difference is simplified as $D=\operatorname{rvec}(\mathbf{Y}) \cdot\left[\left(\mathbf{R}_{Y Y}^{\bar{i}}\right)^{-1}-\left(\mathbf{R}_{Y Y}^{i}\right)^{-1}\right] \cdot[\operatorname{rvec}(\mathbf{Y})]^{H}$, where the determinant term $\operatorname{det}\left(\mathbf{R}_{Y Y}\right)$ in (13) diminishes, as the SNR increases. As a result, the characteristic function of $D$ is given by $\Phi_{D}(s)=\prod_{k=1}^{N_{R} N_{w}} \frac{1}{\lambda_{k} \cdot s+1}$, where $\lambda_{k}$ is the $k$-th eigenvalue of $\mathbf{R}_{Y Y}^{i}\left[\left(\mathbf{R}_{Y Y}^{\bar{i}}\right)^{-1}-\left(\mathbf{R}_{Y Y}^{i}\right)^{-1}\right]$ [41], [42]. A simple approach to the evaluation of this PEP is to firstly formulate the function $f(s)=\frac{1}{s} \prod_{k=1}^{N_{R} N_{w}} \frac{1}{\lambda_{k} \cdot s+1}$ as $f_{p}(s)=\frac{1}{s-\left(-\frac{1}{\lambda_{k}}\right)}$ and $f_{\bar{p}}(s)=\frac{1}{\lambda_{k} \cdot s} \prod_{\forall \lambda_{\bar{k}} \neq \lambda_{k}} \frac{1}{\lambda_{\bar{k}} \cdot s+1}$. Then we apply the Laurent series based representation of $f_{\bar{p}}(s)$, where the constant coefficient associated with index $n=0$ is the residue at the pole $s=-\frac{1}{\lambda_{k}}$, which is given by $f_{\bar{p}}\left(-\frac{1}{\lambda_{k}}\right)=-\prod_{\forall \lambda_{\bar{k}} \neq \lambda_{k}} \frac{\lambda_{k}}{\lambda_{k}-\lambda_{\bar{k}}}$. In summary, the PEP in (40) may be evaluated by:

$$
p\left(\overline{\mathbf{S}}^{i} \rightarrow \overline{\mathbf{S}}^{\bar{i}}\right) \leq \sum_{\forall \lambda_{k}<0} \prod_{\forall \lambda_{\bar{k}} \neq \lambda_{k}} \frac{\lambda_{k}}{\lambda_{k}-\lambda_{\bar{k}}}
$$

Both the theoretical and simulation results portrayed by Fig. 6(a) demonstrate that the HDD-MSDSD aided 16-DQAM schemes significantly outperform its MSDSD aided 16-DPSK counterpart. In Fig. 6(b), the performance advantage of DQAM over DPSK is shown to be as much as $5 \mathrm{~dB}$ and more than $50 \mathrm{~dB}$ at $\mathrm{BER}=10^{-5}$ for the cases of $M=16$ and $M=64$, respectively, when the MSDSD window is increased to $N_{w}=6$. The performance of MSDSD aided 64-DPSK is not shown for $E_{b} / N_{0}>40 \mathrm{~dB}$ in Fig. 6(b), because it is out of scale. This feature verifies that DQAM is especially preferred over DPSK for higher-order modulation schemes.

Furthermore, for the comparison of DQAM constellations, Fig. 6 demonstrates that DAPSK and ADPSK perform similarly in uncoded scenarios, while TADPSK does not provide any noticeable performance improvement, as predicted by Fig. 4(b). Moreover, Fig. 6 also shows that TADPSK ${ }^{\mathrm{JM}}$ performs slightly worse than its DQAM counterparts. This is because the joint amplitude-phase mapping may result in an even more significantly improved iteration gain for TADPSK $^{\mathrm{JM}}$ in coded systems, which implies that TADPSK ${ }^{\mathrm{JM}}$ detection produces a lower extrinsic information $I_{E}$ without a priori information, i.e. at $I_{A}=0$, but the $I_{E}$ achieved by TDAPSK $^{\mathrm{JM}}$ detection may be higher than that of DAPSK and TADPSK in the presence of perfect a priori information, i.e. for $I_{A}=1$. We will continue this discussion in Sec. VIII. 


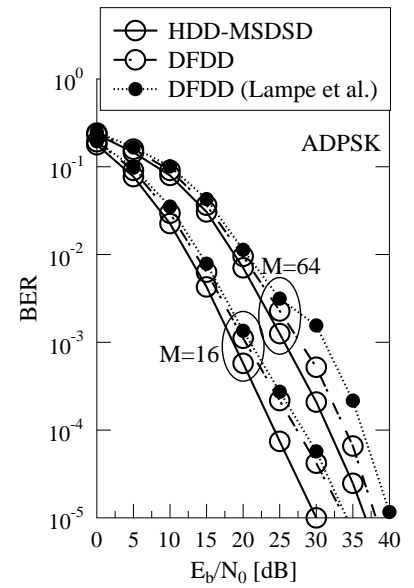

(a)

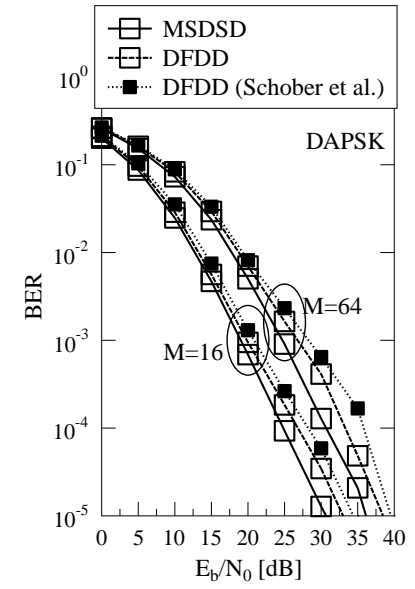

(b)

Fig. 7. BER performance of DFDD aided ADPSK and DAPSK, where we have $N_{w}=6, N_{R}=2$ and $f_{d}=0.03$. The performance of the DFDD conceived for ADPSK (Lampe et al.) in [13] and the DFDD (Schober et al.) conceived for DAPSK in [12] are also portrayed as benchmarks. (a) ADPSK. (b) DAPSK

Fig. 7 portrays the BER performance of DFDD aided ADPSK and DAPSK. As demonstrated in Sec. V, the proposed DFDD is the decision-feedback version of the HDD-MSDD. Therefore, the DFDD is also capable of mitigating the CDD's error floor, when the fading channels fluctuate rapidly, but the HDD-MSDSD still appears to be superior, as confirmed by Fig. 7. Furthermore, as expected, the conventional DFDD solutions of [12], [13], which assumed a constant $\mathbf{C}$ in (15) impose a performance loss, as demonstrated by Fig. 7 .

\section{Complexity Comparison}

The complexity of HDD-MSDD of Sec. III, that of HDDMSDSD of Sec. IV-B invoked by TADPSK ${ }^{\mathrm{JM}}$ as well as that of the reduced-complexity HDD-MSDSD of Sec. IV-C invoked by ADPSK are quantified in terms of the total number of realvalued multiplications in Fig. 8. It can be seen in Fig. 8 that the HDD-MSDSD proposed in Sec. IV substantially reduces the HDD-MSDD complexity, where the HDD-MSDSD complexities converge to their lower bounds, as $E_{b} / N_{0}$ increases. Furthermore, Fig. 8 demonstrates that compared to TADPSK ${ }^{\mathrm{JM}}$ detection, the HDD-MSDSD of Sec. IV-C, which separately visits the ring-amplitude and phase subsets, exhibits a significantly reduced complexity for ADPSK detection, which is also in line with the examples portrayed in Figs. 2 and 3.

Although the MSDD complexity is efficiently reduced by the MSDSD as seen in Fig. 8, the SD complexity still remains an exponential function of constellation size at low SNRs, as demonstrated in [43]. As an alternative, the DFDD of Sec. V imposes a detection complexity that is independent of the SNR, which is also portrayed in Fig. 8. The DFDD complexity is shown to be lower than half of the HDDMSDSD complexity at low SNRs, as evidenced by Fig. 8. However, it is also worth noting that the DFDD complexity is slightly higher than the HDD-MSDSD complexity lower bound seen in Fig. 8. As discussed in Sec. V, the DFDD is equivalent to the HDD-MSDSD at index $v=N_{w}$, which has a higher detection complexity than the HDD-MSDSD at index $v<N_{w}$. Therefore, the average complexity (per symbol) of

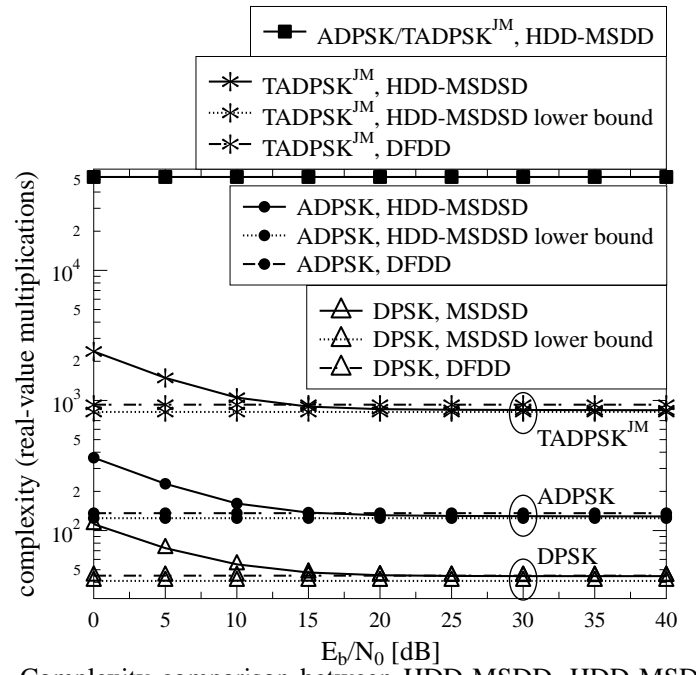

Fig. 8. Complexity comparison between HDD-MSDD, HDD-MSDSD and DFDD aided 16-TADPSK ${ }^{\mathrm{JM}}(2,8), 16-\operatorname{ADPSK}(2,8)$ and 16-DPSK, where we have $N_{w}=3, N_{R}=2$ and $f_{d}=0.03$.

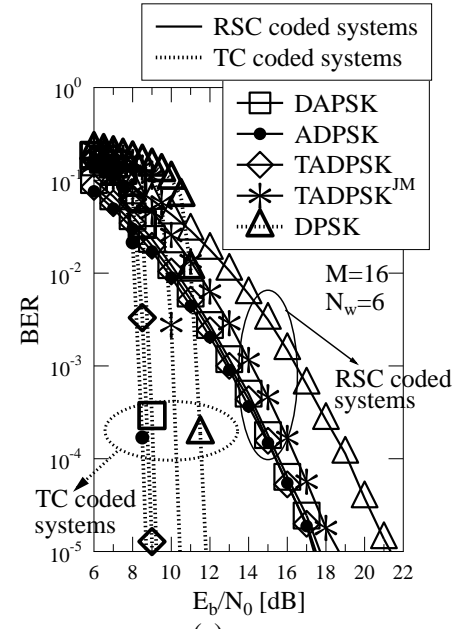

(a)

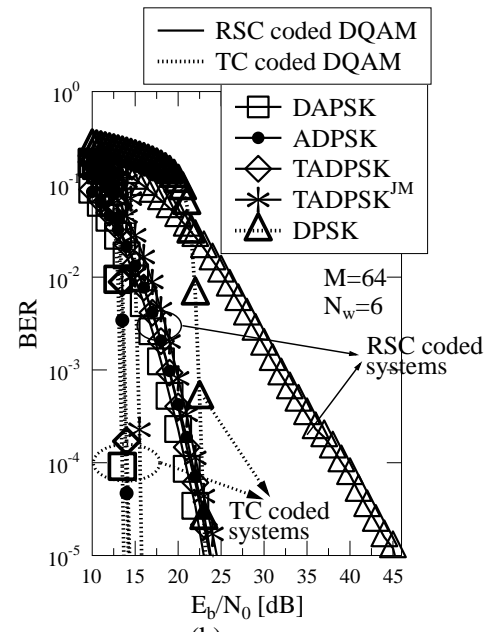

(b)
Fig. 9. BER performance of DAPSK, ADPSK, TADPSK and TADPSK ${ }^{\mathrm{JM}}$ employing HDD-MSDSD as well as DPSK employing MSDSD [18] in RSC and TC coded systems, where we have $N_{R}=2$. There are no iterations between the RSC/TC decoder and the hard-decision HDD-MSDSD/MSDSD. The number of inner iterations between the two RSCs within TC is given by $I R_{T C}=4$. (a) $M=16, N_{w}=6$. (b) $M=64, N_{w}=6$.

DFDD becomes higher than that of the HDD-MSDSD in the high-SNR region.

Moreover, Fig. 8 also demonstrates that as expected, the lowest possible DQAM detection complexities, which correspond to the HDD-MSDSD and DFDD aided 16-ADPSK $(2,8)$ are still about more than $M_{A}=2$ times higher than the 16DPSK detection complexities.

\section{RSC and TC coded DQAM and DPSK}

The performances of HDD-MSDSD aided DQAM schemes are further examined in Recursive Convolutional Code (RSC) and Turbo Code (TC) coded scenarios in Fig. 9. We note that the noncoherent detectors developed in this paper operate based on hard-bit decisions, and hence there are no iterations between the channel decoder and the HDD-MSDSD in Fig. 9. It is demonstrated by Fig. 9 that the different DQAM schemes still perform similarly in RSC and TC coded systems, when the hard-decision HDD-MSDSD is employed. Furthermore, 
Fig. 9 evidences that the HDD-MSDSD aided DQAM schemes also substantially outperform their MSDSD aided DPSK counterparts in coded systems. Explicitly, Figs. 9(a) and (b) demonstrate that in RSC coded systems, HDD-MSDSD aided ADPSK outperforms MSDSD aided DPSK by about $4 \mathrm{~dB}$ and $22 \mathrm{~dB}$ at $\mathrm{BER}=10^{-5}$ for $M=16$ and $M=64$, respectively. Figs. 9(a) and (b) also demonstrate that by employing the more powerful TC, the performance differences between HDDMSDSD aided ADPSK and MSDSD aided DPSK are reduced to about $3 \mathrm{~dB}$ and $8.5 \mathrm{~dB}$ for $M=16$ and $M=64$, respectively, provided that the resultant substantially increased channel decoding complexity is affordable for the specific DQAM system considered.

\section{CONCLUSIONS}

In this paper, we solved the open problem of SD-aided DQAM amplitude detection, so that the MSDSD algorithms that were originally proposed for DPSK [18], [19] become applicable for all DQAM constellations. Our capacity results of Fig. 4 and BER results of Fig. 6 and Fig. 9 demonstrated that the proposed MSDSD aided DQAM schemes significantly outperfom their DPSK counterparts of [18]. Furthermore, a reduced-complexity MSDSD search strategy was also proposed for the DQAM constellations which separately maps bits to ring-amplitude index and phase index, so that the MSDSD complexity imposed for DQAM detection was substantially reduced, as evidenced by Fig. 8. Moreover, we also improved the performance of the conventional DFDD aided DQAM solutions of [12], [13], as demonstrated by Fig. 7. Fig. 8 suggested that the detection complexity of hard-decision MSDSD and DFDD aided DQAM schemes was at least $M_{A}$ times higher than that of their DPSK counterparts.

\section{Further Discussions ON "TURbo DQAM"}

Due to the space limit, we dedicated our efforts to harddecision DQAM detection in this paper, which builds the foundation of offering further solutions to soft-decision DQAM detection. More explicitly, as demonstrated in [19], the softdecision MSDSD exhibits a beneficial iteration gain, which can only be exploited by invoking iterations between the MSDSD and the channel decoder. Although the performance of MSDSD aided DQAM schemes has been examined in coded systems in Sec. VI-D, where the channel decoder is directly concatenated with the hard-decision DQAM detectors, the ultimate goal is to more closely approach to the full performance potential of DQAM constellations promised by the capacity results seen in Sec. VI-A with the aid of turbo detection in channel-coded DQAM systems.

Historically, May et al. [30] proposed Trellis decoded DQAM, and then Fischer et al. [44] proposed to invoke MSDD aided DQAM for BICM in 2001. Moreover, Ishibashi et al. [39] proposed the low-complexity soft-decision CDD aided DAPSK, where the amplitude and phase are separately detected. However, no iterations were invoked between the channel decoder and the DQAM detector in these studies. In 2011, Liang et al. [40] proposed to employ CDD aided DAPSK in turbo detection, where the amplitude and phase are jointly detected. This solution was further streamlined by Xu $e t$ al. [31] in 2013. Furthermore, in 2012, Wang and Hanzo [24] proposed the soft-decision MSDD-IAP aided DAPSK, which was introduced in Sec. I.

As the SD has been invoked for DQAM amplitude detection in this paper, it becomes feasible now to apply the softdecision MSDSD aided DPSK of [19] to DQAM. More explicitly, the PED increment of (26) may be modified for soft-decision MSDSD as:

$$
\begin{aligned}
\Delta_{v-1}=\left\|\widetilde{l}_{1,1} \bar{\Psi}_{v-1}^{*} \bar{\Omega}_{v-1}^{*} \mathbf{Y}_{v}+\omega_{v-1} \psi_{v-1}\left(\sum_{t=1}^{v-1} \widetilde{l}_{v-t+1,1} \bar{\Psi}_{t}^{*} \bar{\Omega}_{t}^{*} \mathbf{Y}_{t}\right)\right\|^{2} \\
+\Xi_{v}-\sum_{\bar{k}_{v}=1}^{\mathrm{BPS}}\left[\widetilde{b}_{\bar{k}_{v}} L_{a}\left(b_{\bar{k}_{v}}\right)-\bar{C}_{a, \bar{k}_{v}}\right]
\end{aligned}
$$

where $\left\{\widetilde{b}_{\bar{k}_{v}}\right\}_{\bar{k}_{v}=1}^{\mathrm{BPS}}$ denote the bit-mapping corresponding to the DQAM constellation point for $x_{v-1}=\gamma_{v-1} \omega_{v-1} \psi_{v-1}$, while $\left\{L_{a}\left(b_{\bar{k}_{v}}\right)\right\}_{\bar{k}_{v}=1}^{\mathrm{BPS}}$ refer to the a priori LLRs gleaned from a channel decoder. Moreover, the constant of $\bar{C}_{a, \bar{k}_{v}}=$ $\frac{1}{2}\left[\left|L_{a}\left(b_{\bar{k}_{v}}\right)\right|+L_{a}\left(b_{\bar{k}_{v}}\right)\right]$ is artificially added in (42) in order to maintain a non-negative ED, as discussed in the context of (17) in [45].

Before extensively examining the DQAM performance in turbo detection assisted coded systems, the complexity of the soft-decision MSDSD of [19] has to be substantially reduced for DQAM constellations, while the potential error propagation problem of HDD-MSDSD has to be avoided. More explicitly, we aim for tackling the following issues for "Turbo DQAM" in our future work. Firstly, the reducedcomplexity soft-decision MSDSD aided DPSK of [45] should be further developed for DQAM, so that the soft-decision SD becomes capable of visiting a reduced number of DQAM constellation points without any performance loss. Secondly, the MSDD-IAP of [24] should be further developed, where the SD should be invoked for amplitude detection, while the reduced-complexity algorithm of [45] should be invoked for phase detection. Last but not the least sophisticated, SoftDecision-Directed (SDD) MSDSD has to be developed for the differential-amplitude DQAM schemes in coded systems, where soft-decision-feedback may be invoked in order to avoid the error propagation problem.

It is also worth noting that although hard-decision HDDMSDSD and DFDD perform similarly, as evidenced by Fig. 7, the potentially erroneous decision feedback tends to degrade the authenticity of the LLRs produced by the soft-decision DFDD, which deteriorates the performance of turbo detection, as the number of iterations increases [45]. For this reason, the MSDSD solutions, which are capable of retaining the full detection capability of MSDD are expected to play a more salient role in soft-decision DQAM detection.

\section{REFERENCES}

[1] W. Weber, "Differential encoding for multiple amplitude and phase shift keying systems," IEEE Trans. Commun., vol. 26, pp. 385-391, Mar. 1978.

[2] R. Fischer, L. Lampe, and S. Calabr, "Differential encoding strategies for transmission over fading channels," Int. J. of Electron. and Commun., vol. 54, no. 1, pp. 59-67, 2000. 
[3] M. Simon, G. Huth, and A. Polydoros, "Differentially coherent detection of QASK for frequency-hopping systems-part I: Performance in the presence of a Gaussian noise environment," IEEE Trans. Commun., vol. 30, pp. 158-164, Jan. 1982.

[4] W. Webb, L. Hanzo, and R. Steele, "Bandwidth efficient QAM schemes for Rayleigh fading channels," IEE Proc. I, Commun., Speech and Vision, vol. 138, pp. 169-175, June 1991.

[5] F. Adachi and M. Sawahashi, "Performance analysis of various 16 level modulation schemes under Rayleigh fading," Electron. Lett., vol. 28, pp. 1579-1581, Aug. 1992.

[6] Y. Chow, A. Nix, and J. McGeehan, "Analysis of 16-APSK modulation in AWGN and Rayleigh fading channel," Electron. Lett., vol. 28, pp. 1608-1610, Aug. 1992

[7] H. Rohling and V. Engels, "Differential Amplitude Phase Shift Keying (DAPSK)-a new modulation method for DTVB," in Proc. of IET Int. Broadcasting Convention, 1995, (IBC 95)., pp. 102-108, Sept. 1995.

[8] H. Leib and S. Pasupathy, "The phase of a vector perturbed by Gaussian noise and differentially coherent receivers," IEEE Trans. Inf. Theory, vol. 34, pp. 1491-1501, Nov. 1988.

[9] R. Schober, W. Gerstacker, and J. Huber, "Decision-feedback differential detection of MDPSK for flat Rayleigh fading channels," IEEE Trans. Commun., vol. 47, pp. 1025-1035, July 1999.

[10] F. Adachi and M. Sawahashi, "Decision feedback differential detection of differentially encoded 16APSK signals," IEEE Trans. Commun. vol. 44, pp. 416-418, Apr. 1996.

[11] R. Schober, W. Gerstacker, and J. Huber, "Decision-feedback differential detection scheme for 16-DAPSK," Electron. Lett., vol. 34, pp. 1812 1813, Sept. 1998.

[12] R. Schober, W. Gerstacker, and J. Huber, "Decision-feedback differential detection based on linear prediction for 16DAPSK signals transmitted over flat Ricean fading channels," IEEE Trans. Commun., vol. 49, pp. 1339-1342, Aug. 2001

[13] L. Lampe and R. Schober, "Low-complexity iterative demodulation for noncoherent coded transmission over Ricean-fading channels," IEEE Trans. Veh. Technol., vol. 50, pp. 1481-1496, Nov. 2001.

[14] D. Divsalar and M. Simon, "Maximum-likelihood differential detection of uncoded and trellis coded amplitude phase modulation over AWGN and fading channels-metrics and performance," IEEE Trans. Commun., vol. 42, pp. 76-89, Jan. 1994.

[15] D. Divsalar and M. K. Simon, "Multiple-symbol differential detection of MPSK," IEEE Trans. Commun., vol. 38, pp. 300-308, Mar. 1990.

[16] S. Wilson, J. Freebersyser, and C. Marshall, "Multi-symbol detection of M-DPSK," in Proc. of IEEE Global Telecommun. Conf. and Exhibition (GLOBECOM'89), pp. 1692-1697 vol.3, Dallas, USA, Nov. 1989.

[17] P. Ho and D. Fung, "Error performance of multiple-symbol differential detection of PSK signals transmitted over correlated Rayleigh fading channels," IEEE Trans. Commun., vol. 40, pp. 1566-1569, Oct. 1992.

[18] L. Lampe, R. Schober, V. Pauli, and C. Windpassinger, "Multiple-symbol differential sphere decoding," IEEE Trans. Commun., vol. 53, pp. 19811985, Dec. 2005.

[19] V. Pauli, L. Lampe, and R. Schober, “"Turbo DPSK” using soft multiplesymbol differential sphere decoding," IEEE Trans. Inf. Theory, vol. 52 pp. 1385-1398, Apr. 2006.

[20] L. Hanzo, O. Alamri, M. El-Hajjar, and N. Wu, Near-Capacity MultiFunctional MIMO Systems: Sphere-Packing, Iterative Detection and Cooperation. John Wiley \& Sons, May 2009.

[21] B. Hassibi and B. Hochwald, "Cayley differential unitary space-time codes," IEEE Trans. Inf. Theory, vol. 48, pp. 1485-1503, June 2002.

[22] C. S. Hwang, S. H. Nam, J. Chung, and V. Tarokh, "Differential space time block codes using nonconstant modulus constellations," IEEE Trans. Signal Process., vol. 51, pp. 2955-2964, Nov. 2003.

[23] S. Sugiura, C. Xu, S. X. Ng, and L. Hanzo, "Reduced-complexity coherent versus non-coherent QAM-aided space-time shift keying," IEEE Trans. Commun., vol. 59, pp. 3090-3101, Nov. 2011.

[24] L. Wang and L. Hanzo, "Low-complexity near-optimum multiplesymbol differential detection of DAPSK based on iterative amplitude/phase processing," IEEE Trans. Veh. Technol., vol. 61, pp. 894-900, Jan. 2012.

[25] V. Pauli, L. Lampe, and J. Huber, "Differential space-frequency modulation and fast 2-D multiple-symbol differential detection for MIMOOFDM," IEEE Trans. Veh. Tech., vol. 57, pp. 297-310, Jan 2008.

[26] S. Cheung and R. Schober, "Differential spatial multiplexing," IEEE Trans. Wireless Commun., vol. 5, pp. 2127-2135, Aug 2006.

[27] L. Wang and L. Hanzo, "Differential interference suppression for SDMA-OFDM based on joint multiple-symbol filtering and detection," IEEE Trans. Veh. Tech., vol. 60, pp. 4656-4662, Nov 2011
[28] X. Dong, N. Beaulieu, and P. Wittke, "Error probabilities of twodimensional $M$-ary signaling in fading," IEEE Trans. Commun., vol. 47, pp. 352-355, Mar. 1999.

[29] L. Lampe and R. Fischer, "Comparison and optimization of differentially encoded transmission on fading channels," in Proc. of Int. Symp. on PowerLine Commun. and its Applicat. (ISPLC'99), 1999.

[30] T. May, H. Rohling, and V. Engels, "Performance analysis of Viterbi decoding for 64-DAPSK and 64-QAM modulated OFDM signals," IEEE Trans. Commun., vol. 46, pp. 182-190, Feb. 1998.

[31] C. Xu, D. Liang, S. X. Ng, and L. Hanzo, "Reduced-complexity noncoherent soft-decision-aided DAPSK dispensing with channel estimation," IEEE Trans. Veh. Technol., vol. 62, pp. 2633-2643, Feb. 2013.

[32] Y. Ma, Q. Zhang, R. Schober, and S. Pasupathy, "Diversity reception of DAPSK over generalized fading channels," IEEE Trans. on Wireless Commun., vol. 4, pp. 1834-1846, July 2005.

[33] R. H. Clarke, "A statistical theory of mobile radio reception," Bell Labs Tech. J., vol. 47, pp. 957-1000, 1968.

[34] C. P. Schnorr and M. Euchner, "Lattice basis reduction: Improved practical algorithms and solving subset sum problems," Math. Programming, vol. 66 , pp. 181-191, 1994.

[35] D. S. Bernstein, Matrix Math.: Theory, Facts, and Formulas (2nd Edition). Princeton University Press, 2009.

[36] C. Studer and H. Bolcskei, "Soft-input soft-output single tree-search sphere decoding," IEEE Trans. Inf. Theory, vol. 56, pp. 4827-4842, Sept. 2010.

[37] U. Fincke and M. Pohst, "Improved methods for calculating vectors of short length in a lattice, including a complexity analysis," Math. of computation, vol. 44, no. 170, pp. 463-471, 1985

[38] M. O. Damen, H. El-Gamal, and G. Caire, "On Maximum-Likelihood detection and the search for the closest lattice point," IEEE Trans. Inf. Theory, vol. 49, pp. 2389-2402, Oct. 2003.

[39] K. Ishibashi, H. Ochiai, and R. Kohno, "Low-complexity bit-interleaved coded DAPSK for Rayleigh-fading channels," IEEE J. on Sel. Areas in Commun., vol. 23, pp. 1728-1738, Sept. 2005.

[40] D. Liang, S. X. Ng, and L. Hanzo, "Soft-decision star-QAM aided BICM-ID," IEEE Signal Process. Letters, vol. 18, pp. 169-172, Jan. 2011.

[41] J. Cavers and P. Ho, "Analysis of the error performance of Trelliscoded modulations in Rayleigh-fading channels," IEEE Trans Commun., vol. 40, pp. 74-83, Jan 1992.

[42] E. Biglieri, G. Caire, and G. Taricco, "Computing error probabilities over fading channels: a unified approach," European Trans. Telecommun., vol. 9, no. 1, pp. 15-25, 1998.

[43] J. Jalden and B. Ottersten, "On the complexity of sphere decoding in digital communications," IEEE Trans. Signal Process., vol. 53, pp. 1474-1484, Apr. 2005 .

[44] R. Fischer, L. Lampe, and S. Muller-Weinfurtner, "Coded modulation for noncoherent reception with application to OFDM," IEEE Trans. Veh. Technol., vol. 50, pp. 910-919, July 2001.

[45] C. Xu, X. Zuo, S. X. Ng, R. G. Maunder, and L. Hanzo, "Reducedcomplexity soft-decision multiple-symbol differential sphere detection," IEEE Trans. Commun., vol. 63, pp. 3275-3289, Sept 2015

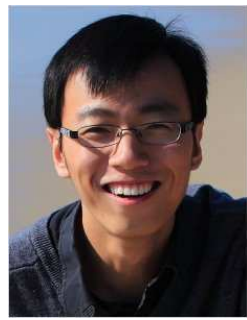

Chao Xu (S'09-M'14) received a B.Eng. degree from Beijing University of Posts and Telecommunications, Beijing, China, and a BSc(Eng) with First Class Honours from Queen Mary, University of London, London, UK, through a Sino-UK joint degree program in 2008, both in Telecommunications Enginneering with Management. He obtained a MSc degree with distinction in Radio Frequency Communication Systems and a Ph.D. degree in Wireless Communications from the University of Southampton, Southampton, UK in 2009 and 2015, respectively. He is currently a postdoctoral researcher working at Southampton Wireless Group, University of Southampton, Southampton, UK. His research interests include reduced-complexity multiple-inputmultiple-output design, noncoherent detection, extrinsic-information-transfer-chart-aided turbo detection, and cooperative communications. He was awarded the Best M.Sc. Student in Broadband and Mobile Communication Networks by the IEEE Communications Society (United Kingdom and Republic of Ireland Chapter) in 2009, and he received 2012 Chinese Government Award for Outstanding Self-Financed Student Abroad. 


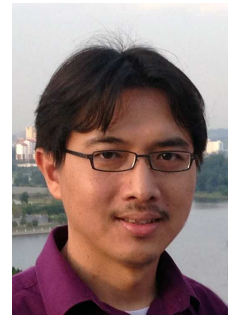

Soon Xin Ng (S'99-M'03-SM'08) received the B.Eng. degree (First class) in electronic engineering and the Ph.D. degree in telecommunications from the University of Southampton, Southampton, U.K., in 1999 and 2002, respectively. From 2003 to 2006, he was a postdoctoral research fellow working on collaborative European research projects known as SCOUT, NEWCOM and PHOENIX. Since August 2006, he has been a member of academic staff in the School of Electronics and Computer Science, University of Southampton. He is involved in the OPTIMIX and CONCERTO European projects as well as the IU-ATC and UC4G projects. He is currently an Associate Professor in telecommunications at the University of Southampton.

His research interests include adaptive coded modulation, coded modulation, channel coding, space-time coding, joint source and channel coding, iterative detection, OFDM, MIMO, cooperative communications, distributed coding, quantum error correction codes and joint wireless-and-optical-fibre communications. He has published over 180 papers and co-authored two John Wiley/IEEE Press books in this field. He is a Senior Member of the IEEE, a Chartered Engineer and a Fellow of the Higher Education Academy in the UK.

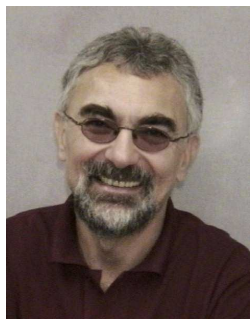

Lajos Hanzo (M'91-SM'92-F'04) FREng, FIEEE, FIET, Fellow of EURASIP, DSc received his degree in electronics in 1976 and his doctorate in 1983. In 2009 he was awarded an honorary doctorate by the Technical University of Budapest, while in 2015 by the University of Edinburgh. During his 38 -year career in telecommunications he has held various research and academic posts in Hungary, Germany and the UK. Since 1986 he has been with the School of Electronics and Computer Science, University of Southampton, UK, where he holds the chair in telecommunications. He has successfully supervised about 100 $\mathrm{PhD}$ students, co-authored 20 John Wiley/IEEE Press books on mobile radio communications totalling in excess of 10000 pages, published $1400+$ research entries at IEEE Xplore, acted both as TPC and General Chair of IEEE conferences, presented keynote lectures and has been awarded a number of distinctions. Currently he is directing a 60-strong academic research team, working on a range of research projects in the field of wireless multimedia communications sponsored by industry, the Engineering and Physical Sciences Research Council (EPSRC) UK, the European Research Council's Advanced Fellow Grant and the Royal Society's Wolfson Research Merit Award. He is an enthusiastic supporter of industrial and academic liaison and he offers a range of industrial courses. He is also a Governor of the IEEE VTS. During 2008 - 2012 he was the Editor-in-Chief of the IEEE Press and a Chaired Professor also at Tsinghua University, Beijing. His research is funded by the European Research Council's Senior Research Fellow Grant. For further information on research in progress and associated publications please refer to http://www-mobile.ecs.soton.ac.uk Lajos has 22 000+ citations. 